\title{
Transient granular shock waves and upstream motion on a staircase
}

\author{
Ko van der Weele, ${ }^{1}$ Giorgos Kanellopoulos, ${ }^{1}$ Christos Tsiavos, ${ }^{1}$ and Devaraj van der Meer ${ }^{2}$ \\ ${ }^{1}$ Department of Mathematics, University of Patras, 26500 Patras, Greece \\ ${ }^{2}$ Physics of Fluids Group, University of Twente, P.O. Box 217, 7500 AE Enschede, The Netherlands \\ (Received 18 February 2009; revised manuscript received 23 May 2009; published 16 July 2009)
}

\begin{abstract}
A granular cluster, placed on a staircase setup, is brought into motion by vertical shaking. Molecular dynamics simulations show that the system goes through three phases. After a rapid initial breakdown of the cluster, the particle stream organizes itself in the form of a shock wave moving down the steps of the staircase. As this wave becomes diluted, it transforms into a more symmetric flow, in which the particles move not only downwards but also toward the top of the staircase. This series of events is accurately reproduced by a dynamical model in which the particle flow from step to step is modeled by a flux function. To explain the observed scaling behavior during the three stages, we study the continuum version of this model (a nonlinear partial differential equation) in three successive limiting cases. (i) The first limit gives the correct $t^{-1 / 3}$ decay law during the rapid initial phase, (ii) the second limit reveals that the transient shock wave is of the Burgers type, with the density of the wave front decreasing as $t^{-1 / 2}$, and (iii) the third limit shows that the eventual symmetric flow is a slow diffusive process for which the density falls off as $t^{-1 / 3}$ again. For any finite number of compartments, the system finally reaches an equilibrium distribution with a bias toward the lower compartments. For an unbounded staircase, however, the $t^{-1 / 3}$ decay goes on forever and the distribution becomes increasingly more symmetric as the dilution progresses.
\end{abstract}

DOI: 10.1103/PhysRevE.80.011305

\section{INTRODUCTION}

Granular flows and avalanches are abundant both in the natural environment and in man-made applications, ranging from giant landslides to the tiny, well-controlled flow of sand grains in an hourglass [1-12]. In this paper, we study the flow of vibrofluidized granular matter through an array of connected compartments in a staircase setup (Fig. 1), being an idealized model for the transport of particles-coal, grain, etc.-along industrial production lines. Given that roughly $4 \%$ of the worldwide energy budget is yearly being wasted due to problems with the handling of granular materials, this is an issue of enormous economical and environmental importance [13-15].

From a more fundamental point of view, it is also a prime example of a many-body system far from equilibrium with a preferential direction, closely related, e.g., to traffic flow on the highway [16-18]. In any such system, the competition between energy input and energy dissipation gives rise to the spontaneous formation of patterns in the flow [19-21]. Traffic jams on the highway are a typical instance and also on the staircase of Fig. 1, one witnesses intriguing flow patterns as a result of the interplay of the energy input (from the vibrations) on the one side and the dissipation (from the inelastic particle collisions) on the other.

These patterns are discussed in Sec. II. By means of molecular dynamics (MD) simulations, we follow the time evolution of a cluster of particles placed near the top of the vibrated staircase. We observe three successive flow regimes: an initial fast breakdown of the cluster, followed by the formation of a shock wave running down the stairs, which eventually transforms into a more symmetric diffusive flow with particles moving both upward and downward. Given a long but finite staircase, the system finally settles into a diluted and nearly homogeneous distribution (biased toward the lower steps) in which there is a dynamic equilibrium between any two neighboring compartments. On an infinitely
PACS number(s): 45.70.- $-\mathrm{n}, 05.60 .-\mathrm{k}, 02.60 . \mathrm{Lj}$

long staircase, the diffusive process would continue forever.

To analyze this behavior, in Sec. III, we set up a theoretical model, in which the particle flow from one compartment to the next is represented by a flux function depending on the number of particles in the compartment and the shaking strength. The flow patterns obtained from this flux model are in good agreement with the MD simulations. In order to explain the underlying physics of the process, in Sec. IV, we turn to the continuum version of the model, where the position along the staircase is no longer treated as a discrete variable but as a continuous one. The flux balance then takes the form of a nonlinear partial differential equation in which terms of first and second order in $\Delta x$ (the width of the steps) compete for dominance. This is worked out further in Sec. V. (i) The rapid initial stage is dominated by the second-order terms and the $t^{-1 / 3}$ decay is shown to be a direct consequence of this. (ii) The shock wave behavior is dominated by the first-order terms and the associated density decay goes as $t^{-1 / 2}$. (iii) Third, the diffusive regime is dominated by the second-order terms again and the density falls off as $t^{-1 / 3}$. In Sec. VI, we comment on the role of the shaking strength and demonstrate that the transient shock wave only shows up when the shaking is not too strong; otherwise one witnesses a direct transition to the diffusive behavior. A general conclusion is given in Sec. VII. The paper is accompanied by a mathematical Appendix, in which we analyze the differential equation that governs the approximate self-similarity of the flow during the stages (i) and (iii).

\section{MD SIMULATIONS}

In our MD simulations, we use a three-dimensional eventdriven code. Between two events (collisions), the particles move freely, describing parabolic paths under the influence of gravity, until the next collision occurs. A collision can be either between particles or between a particle and a wall. At 


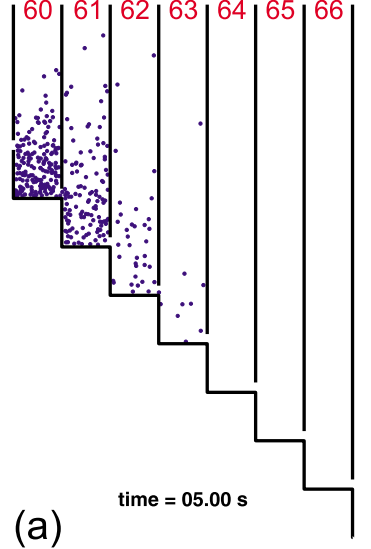

(b)

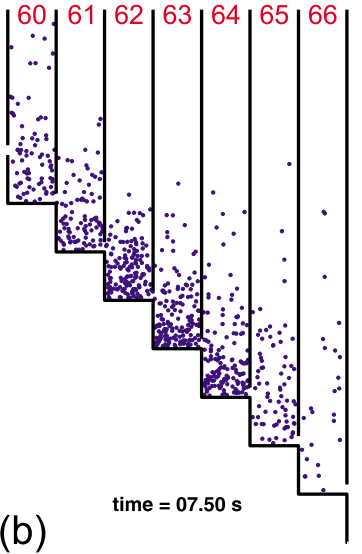

(c)

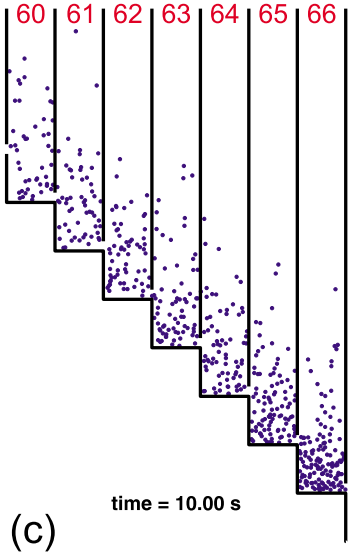

(d)

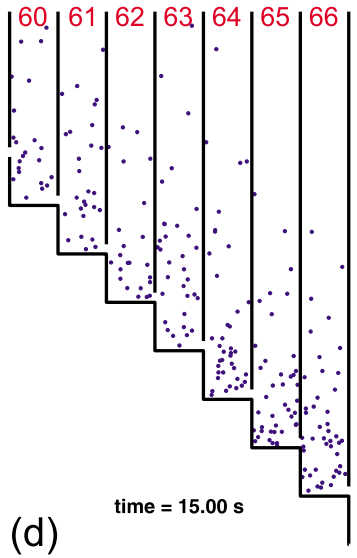

FIG. 1. (Color online) Four snapshots of a partial view of the staircase system, showing compartment 60-66 of the 1000 connected compartments used in the MD simulations [22]. The setup is vibrated in the vertical direction at frequency $f=100 \mathrm{~Hz}$ and amplitude $a$ $=0.2 \mathrm{~mm}$, causing the particles to form a granular gas. At $t=0 \mathrm{~s}$ (not shown), the experiment starts with 1000 particles distributed equally over compartments 50 and 51. (a) At $t=5.00 \mathrm{~s}$, the downward-moving particle front has reached the first of the displayed compartments. (b) At $t=7.50 \mathrm{~s}$, it is traveling further to the right. (c) At $t=10.00 \mathrm{~s}$, the front has just passed through compartment 66. (d) Afterwards, as exemplified by the snapshot at $t=15.00 \mathrm{~s}$, it leaves a trail of particles which becomes more dilute as time progresses.

any such event, the velocities of the particles after contact are computed from the velocities just before contact using Newton's laws. The particles are hard spheres with a coefficient of normal restitution (for particle-particle collisions) that is taken to be constant, $e=0.95$. The coefficients of tangential restitution and dynamical friction are set equal to their ideal, dissipationless values. Likewise, the collisions with the walls and bottom are taken to be elastic.

The ground area of each compartment is $\Omega=25$ $\times 25 \mathrm{~mm}^{2}$, the size of the rectangular opening between the compartments is $S=5 \times 25 \mathrm{~mm}^{2}$, and the step height is $h_{\text {step }}=25 \mathrm{~mm}$. The simulated system has a length of $K$ $=1000$ compartments [22]. The whole setup is vibrated vertically with adjustable frequency $f$ and amplitude $a$ following a sinusoidal wave form.

Figures 1 and 2 show the MD results for $N_{\text {tot }}=1000$ beads of diameter $d=1 \mathrm{~mm}$, initially distributed evenly over compartments 50 and 51, vibrated at an amplitude $a=0.2 \mathrm{~mm}$ and frequency $100 \mathrm{~Hz}$. The cluster is seen to dissolve quickly into a downward rush of particles, which organizes itself in the form of a shock wave, recognizable from its characteristic dense front [see Fig. 1 and the profiles from $t=4.0 \mathrm{~s}$ and onward in Fig. 2(a)]. The density and speed of this wave gradually diminish as it travels toward the right. After a certain time, when the flow has become quite dilute, it becomes more symmetric toward both sides. The particles do not only run downwards anymore but are also frequently seen to jump uphill, against gravity, like trout swimming upriver. This is illustrated in Fig. 2(b), where we see that the density profile in the long-time limit develops a considerable tail in the compartments $1-49$, i.e., to the left of the original cluster. In fact, there is a clear heaping effect visible in the leftmost compartments.

In Fig. 2(c), the measured height of the maximum in the density profile $\left(n_{\max }\right)$ is plotted as a function of time. This is a measure of how fast the flow dilutes. Three successive regimes can be discerned: (i) the initial breakdown of the cluster, during which $n_{\max }(t)$ decays as $t^{\alpha}$, with $\alpha=-0.33$, (ii) the shock wave, decaying in height as $t^{\beta}$, with $\beta=-0.63$, and (iii) the eventual symmetric flow during which the profile decays as $t^{\gamma}$, with $\gamma=-0.31$. The crossovers between these stages, at $\log _{10} t \approx 0.8(t \approx 6.3 \mathrm{~s})$ and $\log _{10} t \approx 2(t \approx 100 \mathrm{~s})$, respectively, are seen to be quite sharp. All the above observations will be rationalized-qualitatively and quantitatively - within the context of a dynamical flux model that we introduce in the next section.

\section{FLUX MODEL}

The flux model we employ is based on the model that was used earlier to describe the dynamics of granular material in a nontilted array of compartments [23-29]. At the heart of this model is the so-called flux function $F_{L, R}\left(n_{k}\right)$, which gives the outflow from compartment $k$ (to its left and right neighbors, respectively) as a function of the fraction of particles contained in it. If $N_{k}(t)$ denotes the number of particles in compartment $k$ at time $t$ and $N_{\text {tot }}$ the total number of particles in the system, then $n_{k}(t)=N_{k}(t) / N_{\text {tot }}$.

In a horizontal system, the flux functions to both sides are equal. In the staircase setup, however, the height difference between the left and right apertures (see Fig. 1) produces a bias toward the right. The general form of the flux function is $[23,24]$

$$
F_{L, R}\left(n_{k}\right)=A n_{k}^{2} e^{-B_{L, R} n_{k}^{2}},
$$

where the fraction $n_{k}(t)$ is subject to the condition $\sum_{k=1}^{K} n_{k}(t)=1$, with $K$ the number of compartments. In combination with the fact that $N_{\text {tot }}$ is constant, this expresses the mass conservation in the system.

The factor $A$, which determines the absolute rate of the flux, is given by [28]

$$
A \propto\left(1-e^{2}\right)^{2} \frac{N_{\mathrm{tot}} r^{2} S}{\Omega^{2}} \frac{g}{a f},
$$

with $g=9.81 \mathrm{~m} / \mathrm{s}^{2}$ the gravitational acceleration and the precise value of the proportionality constant being dependent on 

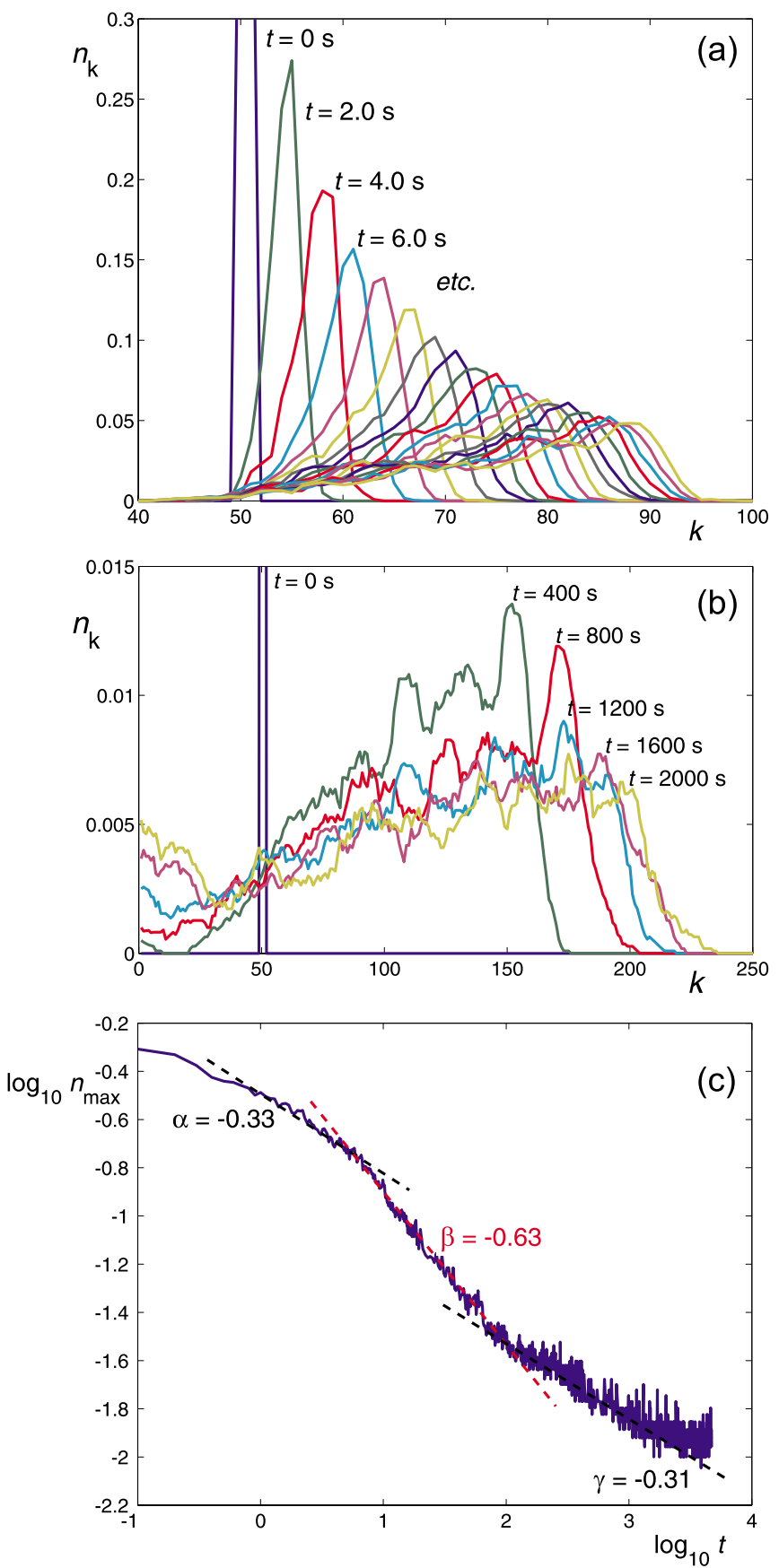

FIG. 2. (Color online) (a) The particle distribution $n_{k}(t)$ obtained from MD simulations with 1000 particles at 20 successive times starting from a cluster in compartments 50 and $51\left[n_{50}(0)=n_{51}(0)\right.$ $=0.50$, while the remainder of the $K=1000$ compartments is initially empty], at constant intervals of $2.0 \mathrm{~s}$. The shaking parameters are the same as in Fig. 1. (b) The particle distribution $n_{k}(t)$ on a much longer time scale at intervals of $400 \mathrm{~s}$. To reduce noise, a running average over 21 compartments has been taken (except for the initial distribution at $t=0 \mathrm{~s})$. Note that a substantial-and increasing - amount of granular matter flows upwards, toward the left of compartment 50. (c) Doubly logarithmic plot of the maximum of the particle distribution $\left[n_{\max }(t)\right]$ vs time. The three successive regimes (initial breakdown, shock wave, and symmetric diffusion) are indicated by dashed straight lines with slopes -0.33 , -0.63 , and -0.31 , respectively. the details of the driving. There is no distinction between left and right as far as this factor $A$ is concerned, which stands to reason, since in the dilute limit $n_{k} \rightarrow 0$ the flux functions $F_{L}\left(n_{k}\right) \rightarrow A n_{k}^{2}$ and $F_{R}\left(n_{k}\right) \rightarrow A n_{k}^{2}$ must become equal. This is because the decrease in density over the step height $h_{\text {step }}$ becomes negligible in this limit. The factor $A$ may be incorporated in the time scale $t$ by introducing a dimensionless time variable $\tau=A t$. We will adopt a similar strategy by keeping the original time $t$ but taking $A=1 \mathrm{~s}^{-1}$ in all our flux model calculations.

The dimensionless parameter $B$ is given by (with the precise proportionality constant again depending on the driving) [28]

$$
B_{L, R} \propto\left(1-e^{2}\right)^{2}\left[\frac{N_{t o l} r^{2}}{\Omega}\right]^{2} \frac{g h_{L, R}}{(a f)^{2}} .
$$

This parameter $B_{L, R}$ is the product of three dimensionless parts with a clear physical interpretation: (i) the factor $\left(1-e^{2}\right)^{2}$ (equal to 0.010 for $e=0.95$ ), where $\left(1-e^{2}\right)$ represents the fraction of the center-of-mass kinetic energy that is lost in a single collision between any two particles, (ii) the filling factor $r^{2} N_{\text {tot }} / \Omega$ squared, and (iii) the ratio between the energy needed for a particle to overcome the barrier $\left(m g h_{L . R}\right)$ and the typical kinetic energy conveyed to the particles by the vibrating setup $\left[\propto m(a f)^{2}\right]$.

The only difference between the particle fluxes to the left and right lies in the barrier height $h_{L, R}$. For jumps toward the right, this height is zero $\left(h_{R}=0\right)$, so $B_{R}=0$, whereas for jumps toward the left it is equal to the step height $\left(h_{L}=h_{\text {step }}\right)$. This gives

$$
\begin{gathered}
F_{R}\left(n_{k}\right)=A n_{k}^{2}, \\
F_{L}\left(n_{k}\right)=A n_{k}^{2} e^{-B_{L} n_{k}^{2}} .
\end{gathered}
$$

These two flux functions have been depicted in Fig. 3 for $A=1 \mathrm{~s}^{-1}$ and $B_{L}=1000$; this value of $B_{L}$ roughly corresponds to the choice of parameters used in the MD simulations of Fig. 2. Both $F_{R}\left(n_{k}\right)$ and $F_{L}\left(n_{k}\right)$ start out from zero at $n_{k}=0$ (if there are no particles in the compartment, it can give nothing to any of its neighbors) and in the limit $n_{k} \rightarrow 0$, the two fluxes are equal. This at once explains the observation that the flow becomes increasingly symmetric toward both sides in the long-time limit when all compartments are diluted.

We see that $F_{R}\left(n_{k}\right)$ is a monotonically increasing function of $n_{k}$ : the more particles a compartment contains, the more it will give to its right-hand neighbor. In contrast, the function $F_{L}\left(n_{k}\right)$ shows a maximum: at small particle numbers, it increases with $n_{k}$, but beyond $n_{k}=1 / \sqrt{B_{L}}$ the increasingly frequent (inelastic) collisions make the particles in the compartment so slow that they are hardly able to overcome the step height anymore and the flux starts to decrease. For large $n_{k}$, the function $F_{L}\left(n_{k}\right)$ goes to zero again.

In contrast to the horizontal system studied by Eggers and others [23-29], our staircase does not have the tendency to form any clustered states with particles clogging together in one compartment, not even at very mild shaking strengths. This is a consequence of our choice $h_{R}=0 \mathrm{~mm}$ (or $B_{R}=0$ ). The stabilization of a cluster would require a flux balance 


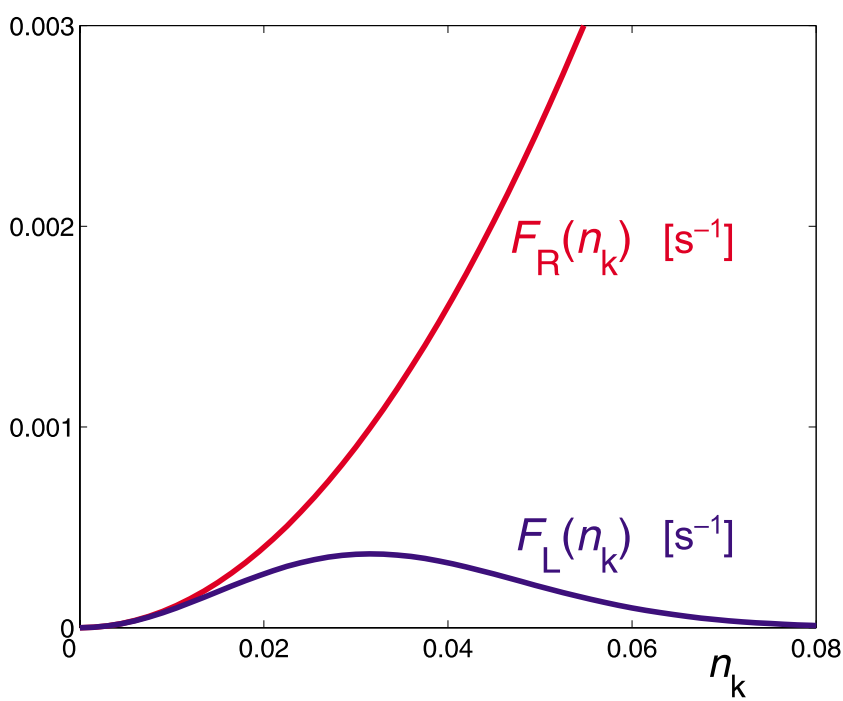

FIG. 3. (Color online) The flux functions toward the right $\left[F_{R}\left(n_{k}\right)\right.$, Eq. (4)] and the left $\left[F_{L}\left(n_{k}\right)\right.$, Eq. (5)] as function of $n_{k}$, i.e., the fraction of particles in the $k$ th compartment. In the dilute limit $n_{k} \rightarrow 0$, the fluxes start out equal, but for larger $n_{k}$, the difference between them rapidly diverges. In this plot, we have taken $A$ $=1 \mathrm{~s}^{-1}$ and $B_{L}=1000$.

between the well-filled compartment and the two diluted ones around it (separately with each one of them), but this could only be accomplished if not only $F_{L}\left(n_{k}\right)$ but also $F_{R}\left(n_{k}\right)$ were nonmonotonic, i.e., if the height $h_{R}$ were nonzero. We have intentionally ruled out this possibility because our interest here lies in the flow of particles, not in their clustering.

Given the above flux functions (4) and (5), the evolution of the system is governed by the following balance equation, which expresses the time rate of change of the fraction in compartment $k$ :

$$
\begin{gathered}
\frac{d n_{k}}{d t}=F_{R}\left(n_{k-1}\right)-F_{L}\left(n_{k}\right)-F_{R}\left(n_{k}\right)+F_{L}\left(n_{k+1}\right), \\
k=2,3, \ldots, K-1 .
\end{gathered}
$$

This equation holds for all compartments except the first and the last one. For the first one $(k=1)$, we must suppress the terms $F_{R}\left(n_{k-1}\right)$ and $F_{L}\left(n_{k}\right)$, representing inflow from and outflow toward the left neighbor, and for the last compartment $(k=K)$, we must likewise suppress the terms $F_{R}\left(n_{k}\right)$ and $F_{L}\left(n_{k+1}\right)$. The phenomena discussed in this paper are only marginally affected by the finite size of the system. Indeed, it is for this reason that we take a large number of compartments $(K=1000)$ and choose to position the initial cluster far away from the boundaries [22].

It should be noted that Eq. (6) takes into account only particle jumps between neighboring compartments, in accordance with the fact that the compartments are divided by walls leaving only a narrow slit near the bottom. This prevents jumps to the next-nearest neighbors or beyond. Further, the model does not include any statistical fluctuations (which would introduce a Gaussian white-noise term in Eq. (6)
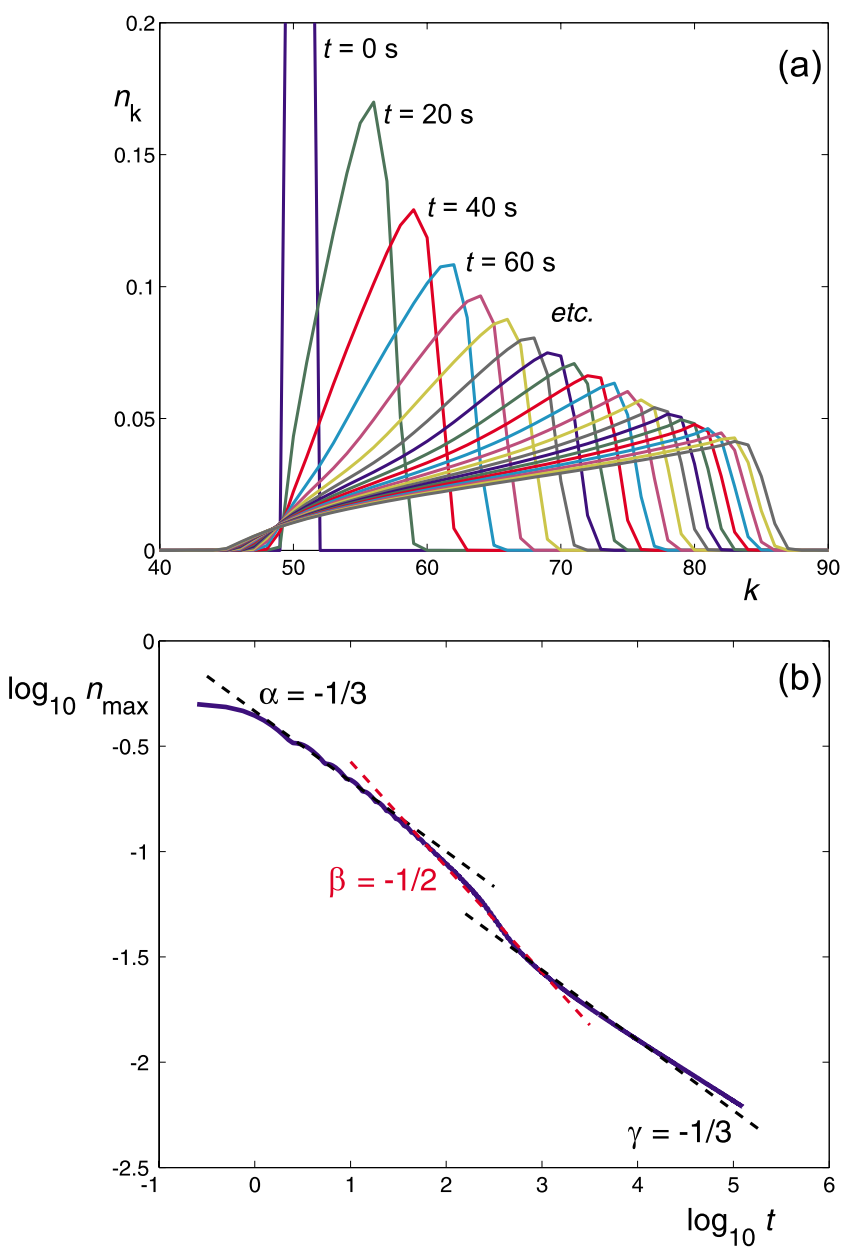

FIG. 4. (Color online) (a) The particle distribution $n_{k}(t)$ calculated with the flux model Eq. (6) at successive times $t$ $=0,20,40, \ldots, 400 \mathrm{~s}$ starting from a cluster in compartments 50 and $51\left[n_{50}(0)=n_{51}(0)=0.50\right]$, while all the other of the $K=1000$ compartments are initially empty. The shaking parameters are $B_{L}$ $=1000$ and $A=1 \mathrm{~s}^{-1}$. (b) Doubly logarithmic plot of the maximum of the particle distribution $\left[n_{\max }(t)\right]$ vs time. Indicated are the initial breakdown stage (characterized by a slope $-1 / 3$ ), the Burgers regime (with slope $-1 / 2$ ), and the diffusive regime (slope $-1 / 3$ ). The oscillatory structures visible for small $t$ reflect the filling and subsequent emptying of successive compartments that are, each in its turn, the best filled one; at the cusps, the maximum jumps from one compartment to the next.

$[23,30])$, so it must be interpreted as a mean-field description of the dynamics, corresponding to an ensemble average of a large number of MD simulations starting from a given initial condition.

How well does the flux model reproduce the MD results? In Fig. 4(a), we show the density profiles calculated from Eq. (6) for $B_{L}=1000$, corresponding to the MD simulations of Fig. 2, and with the same initial condition. The flow according to the flux model is seen to go through the same three stages as the MD simulations: (i) first the fast breakdown of the cluster, followed by (ii) the buildup of a shock wave with a steep front traveling toward the right, and eventually (iii) a more symmetric spreading of the particles when the flow becomes diluted. 
In Fig. 4(b), we plot the height of the maximum of the particle distribution as a function of $t$, just as we did for the MD simulations in Fig. 2(b). We see that the first stage, which lasts up to $\log _{10} t \approx 1.6(t \approx 40 \mathrm{~s})$, is consistent with a density decay $n_{\max }(t) \propto t^{-1 / 3}$, meaning that the data fall on a straight line with slope $-1 / 3$. During the second stage, lasting until some point between $\log _{10} t=2.4$ and $3(t \approx 250 \mathrm{~s}$ to $1000 \mathrm{~s}$, depending on the eye of the beholder), the data agree with a $t^{-1 / 2}$ decay, i.e., slope $-1 / 2$. Afterwards, in the third, dilute stage we return to a $t^{-1 / 3}$ decay with slope $-1 / 3$. The remainder of this paper is largely devoted to the justification of the above exponents.

\section{CONTINUUM VERSION OF THE FLUX MODEL}

\section{A. Reformulating the model with a continuous spatial coordinate}

The physics of the flow process, including the exponents observed in Figs. 2(b) and 4(b), is best understood in terms of the continuum version of the flux model. A similar approach was followed for the nontilted system in Ref. [26].

In the continuum description, the discrete position variable $k$ (indicating the compartments) is replaced by a continuous variable $x$ and hence the fraction $n_{k}(t)$ is replaced by $n(x, t) \Delta x$, where $n(x, t)$ is the number density per unit length and $\Delta x$ the width of a compartment. The continuum counterpart of the conservation condition $\Sigma_{k=1}^{K} n_{k}=1$ is

$$
\int_{0}^{K \Delta x} n(x, t) d x=1 \text {. }
$$

The above replacement of $n_{k}(t)$ by $n(x, t) \Delta x$ also needs to be done in the balance Eq. (6) and for this purpose, it is convenient to introduce the continuum counterparts $\widetilde{F}_{L, R}[n(x, t)]$ of the flux functions $F_{L, R}\left(n_{k}\right)$ as follows:

$$
\widetilde{F}_{L, R}[n(x, t)] \equiv \tilde{A} n^{2} e^{-\tilde{B}_{L, R^{n}} n^{2}}=\frac{1}{\Delta x} F_{L, R}[n(x, t) \Delta x],
$$

which means that $\widetilde{A}=A \Delta x$ and $\widetilde{B}=B \Delta x^{2}$. With Eq. (8), the balance equation Eq. (6) takes the following form:

$$
\begin{aligned}
\frac{\partial n(x, t)}{\partial t}= & \widetilde{F}_{R}[n(x-\Delta x, t)]-\widetilde{F}_{R}[n(x, t)] \\
& -\widetilde{F}_{L}[n(x, t)]+\widetilde{F}_{L}[n(x+\Delta x, t)] \\
= & -\Delta x\left(\frac{\partial \widetilde{F}_{R}}{\partial x}-\frac{\partial \widetilde{F}_{L}}{\partial x}\right) \\
& +\frac{1}{2}(\Delta x)^{2}\left(\frac{\partial^{2} \widetilde{F}_{R}}{\partial x^{2}}+\frac{\partial^{2} \widetilde{F}_{L}}{\partial x^{2}}\right)+\cdots,
\end{aligned}
$$

where the second step follows from a Taylor expansion up to second order in $\Delta x$. Using partial differentiation $\left[\partial \widetilde{F}_{R} / \partial x\right.$ $=\left(d \widetilde{F}_{R} / d n\right)(\partial n / \partial x)$, etc. $]$ this can be written as

$$
\frac{\partial n(x, t)}{\partial t}=-P(n) \frac{\partial n}{\partial x}+\frac{\partial}{\partial x}\left[Q(n) \frac{\partial n}{\partial x}\right]
$$

with the first- and second-order prefactors given by

$$
\begin{aligned}
& P(n) \equiv \Delta x\left(\frac{d \widetilde{F}_{R}}{d n}-\frac{d \widetilde{F}_{L}}{d n}\right)=2 A_{1} n\left[1-\left(1-\widetilde{B}_{L} n^{2}\right) e^{-\widetilde{B}_{L} n^{2}}\right], \\
& Q(n) \equiv \frac{1}{2} \Delta x^{2}\left(\frac{d \widetilde{F}_{R}}{d n}+\frac{d \widetilde{F}_{L}}{d n}\right)=A_{2} n\left[1+\left(1-\widetilde{B}_{L} n^{2}\right) e^{-\widetilde{B}_{L} n^{2}}\right] .
\end{aligned}
$$

Here, we have used the expressions for the flux functions $\widetilde{F}_{R, L}$ given by Eq. (8). Further, we have defined $A_{1}=\tilde{A} \Delta x$ $=A \Delta x^{2}$ and $A_{2}=\tilde{A} \Delta x^{2}=A \Delta x^{3}$.

The partial differential equation (10) is the continuum counterpart of the balance equation Eq. (6). If the prefactor $P(n)$ in the first-order term $-P(n) \partial n / \partial x$ would be simply a positive constant, this term would be associated with a translation to the right of the density profile, with wave velocity $P$. Likewise, if the factor $Q(n)$ in the second-order term would be a positive constant, this term would reduce to an ordinary diffusive term $Q \partial^{2} n / \partial x^{2}$, tending to smoothen the profile. However, although the factors $P(n)$ and $Q(n)$ are both positive for $n>0$, they are not constant and hence the behavior of Eq. (10) is richer and more complicated than in the case with constant coefficients.

A word about the dimensionality of the problem is in order here. By replacing the dimensionless variable $k$ by the variable $x$, which has the dimension of length $([x]=L)$, and the dimensionless fraction $n_{k}(t)$ by the number density per unit length $n(x, t)$ (dimension $[n]=1 / L$ ), the various parameters in the model have acquired a different dimensionality too $\left[\widetilde{B}_{L}\right]=L^{2}$ and the dimensions of $A_{1}$ and $A_{2}$ can be inferred immediately from their definitions below Eq. (12)

$$
\left[A_{1}\right]=\frac{L^{2}}{T}, \quad\left[A_{2}\right]=\frac{L^{3}}{T} .
$$

For the sake of simplicity, we choose $\Delta x=1$, which means (together with our earlier choices $A=1 \mathrm{~s}^{-1}$ and $B_{L}=1000$ ) that the parameters in the continuum model have the numerical values $\widetilde{B}_{L}=1000 \mathrm{~m}^{2}, A_{1}=1 \mathrm{~m}^{2} / \mathrm{s}$, and $A_{2}=1 \mathrm{~m}^{3} / \mathrm{s}$. Conveniently, it also means that we may identify the density profiles $n(x, t)=n_{k}(t)$, with $x=k \Delta x=k$ meter [31].

\section{B. Changing dominance of the first- and second-order terms}

In order to analyze the successive flow regimes, it is of key importance to evaluate the relative magnitude of the first- and second-order terms in the balance equation (10). To obtain an estimate for this quantity, we first note that $\partial n / \partial x$ is of order $n_{\max } / \ell$, where $\ell$ denotes the half width of the density profile. Since $\ell n_{\max }$ is proportional to the total area under the profile (which is equal to 1 ), we may set $\ell \sim 1 / 2 n_{\max }$. Thus,

$$
\frac{\partial n}{\partial x} \approx \gamma n_{\max }^{2},
$$

with $\gamma$ a numerical prefactor in the order of 2 . With this, the estimated magnitude of the first-order term becomes

$$
P(n) \frac{\partial n}{\partial x} \approx \gamma P\left(n_{\max }\right) n_{\max }^{2} .
$$

In the same approximation, we have $Q(n) \partial n / \partial x$ $\approx \gamma Q\left(n_{\max }\right) n_{\max }^{2}$ and this gives for the second-order term 


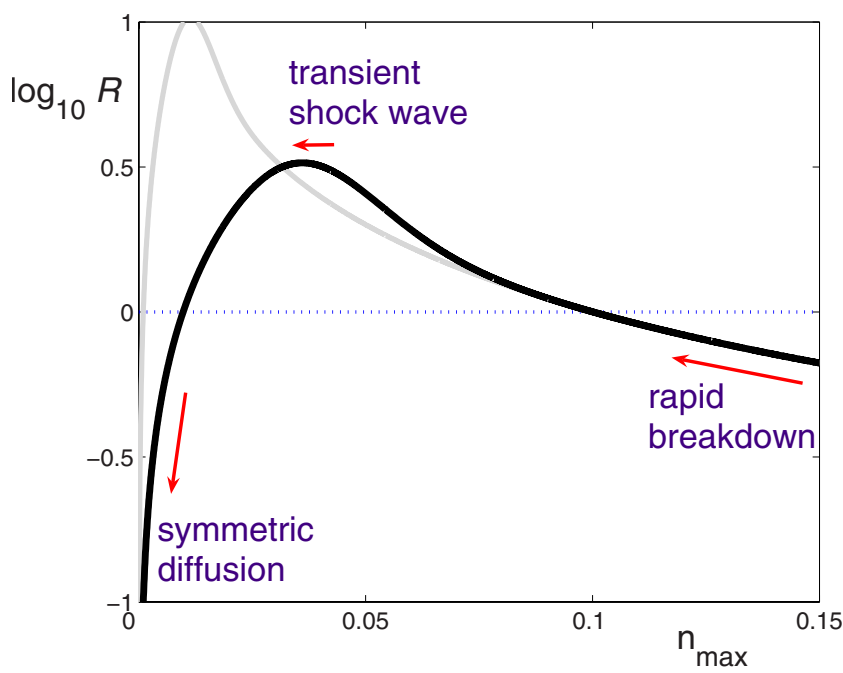

FIG. 5. (Color online) Logarithm of the estimated ratio $R$ between the first- (shock wave) and second-order (diffusive) terms in Eq. (10), as a function of the profile height $n_{\max }$ for $\widetilde{B}_{L}=1000 \mathrm{~m}^{2}$ and $\gamma^{\prime}=20$ (solid black line, corresponding to the MD simulations of Fig. 2 and the flux model results of Fig. 4). For comparison, the gray curve represents the case for $\widetilde{B}_{L}=10000 \mathrm{~m}^{2}$ and $\gamma^{\prime}=20$. At this higher $\widetilde{B}_{L}$ value, the first-order term has gained in importance. The range of $n_{\max }$ for which $\log _{10} R\left(n_{\max }\right)>1$ increases, as well as the magnitude of $R$ itself, i.e., the shock wave lives longer and is also more pronounced.

$$
\frac{\partial}{\partial x}\left(Q(n) \frac{\partial n}{\partial x}\right) \sim \frac{\left[Q(n) \frac{\partial n}{\partial x}\right]_{n_{\max }}}{\ell},
$$

where again $\ell \sim 1 / 2 n_{\max }$ leading to

$$
\frac{\partial}{\partial x}\left(Q(n) \frac{\partial n}{\partial x}\right) \approx \gamma^{\prime} \gamma Q\left(n_{\max }\right) n_{\max }^{3},
$$

where $\gamma^{\prime}$ is a second prefactor larger than 1 .

The relative importance of the first- and second-order terms is given by the ratio $R\left(n_{\max }\right)$ of Eqs. (15) and (17)

$$
R\left(n_{\max }\right) \equiv \frac{P(n) \frac{\partial n}{\partial x}}{\frac{\partial}{\partial x}\left[Q(n) \frac{\partial n}{\partial x}\right]} \approx \frac{1}{\gamma^{\prime}} \frac{P\left(n_{\max }\right)}{n_{\max } Q\left(n_{\max }\right)} .
$$

The logarithm of this ratio is plotted in Fig. 5 for $\widetilde{B}_{L}$ $=1000 \mathrm{~m}^{2}$ and $\gamma^{\prime}=20$. When $\log _{10} R$ is positive, the firstorder term dominates and we get shock wave behavior, as we will explain in the next section.

If on the other hand $\log _{10} R$ is negative, the second-order terms govern the behavior of the system. As we see in Fig. 5, this is the case for $n_{\max }>0.10$ (the initial breakdown stage) and also for $n_{\max }<0.01$ (the symmetric diffusion at large times). These values are in good agreement with the observed transitions in Figs. 2(c) and 4(b).

In the next section, we will concentrate on the dominant terms in the three successive flow regimes, neglecting any other terms, and solve the associated simplified balance equations. As we will see, this not only gives us a clear picture of the physical processes at work but also yields the proper decay exponents.

\section{ANALYSIS OF THE SUCCESSIVE FLOW REGIMES}

\section{A. Initial rapid breakdown of the cluster}

During the initial breakdown of the cluster, the dominant behavior is given by the second-order terms in the balance equation (10). In our analysis, we will simply ignore the term $-P(n) \partial n / \partial x$. Further, at this stage $\widetilde{B}_{L} n^{2} \gg 1$ and so the expression for $Q(n)$ reduces to $A_{2} n$. The balance equation then takes the approximate form

$$
\frac{\partial n}{\partial t}=A_{2} \frac{\partial}{\partial x}\left(n \frac{\partial n}{\partial x}\right) .
$$

This is a nonlinear heat equation [32], differing from the usual (linear) heat equation $\partial n / \partial t=D \partial^{2} n / \partial x^{2}$ by the extra $n$ in the right-hand term.

During the initial stage, the flow is directed almost exclusively toward the right, since $F_{R}(n) \gg F_{L}(n)$ (see Fig. 3). Indeed, the original discrete flux model Eq. (6) at this stage reduces in good approximation to

$$
\frac{d n_{k}}{d t}=F_{R}\left(n_{k-1}\right)-F_{R}\left(n_{k}\right)
$$

This asymmetry is however not reflected by the nonlinear heat equation Eq. (19) (on the contrary, this equation is invariant under the transformation $x \rightarrow-x$, which means that it is completely symmetric with respect to left and right), so we have to impose the asymmetry ourselves as an extra condition. That is, from the family of solutions to the partial differential equation Eq. (19), we must choose a sufficiently asymmetric one.

The observed scaling behavior during the initial stages in Figs. 2(b) and 4(b) (i.e., the density falling off as $t^{-1 / 3}$ ) suggests that we should try a self-similarity solution [33]. On dimensional grounds [34] such a solution must be of the form

$$
n(x, t)=c_{b r}\left(A_{2} t\right)^{-1 / 3} H(\xi),
$$

where the dimensionless function $H(\xi)$ depends on $x$ and $t$ only through the combined dimensionless variable $\xi=\left(x-x_{0}\right)\left(A_{2} t\right)^{-1 / 3}$. The prefactor $c_{b r}$ is a free constant, which we choose to be $c_{b r}=2$; the subscript $b r$ stands for breakdown. As we will see later, this choice makes the present analysis also applicable to stage (iii), i.e., the long-time limit when the system shows diffusive behavior.

Note that from Eq. (21), it immediately follows that the profile height decreases as $n_{\max }(t) \propto t^{-1 / 3}$. Correspondingly, its width increases as $t^{1 / 3}$ because the area under the profile remains constant $(=1)$ in accordance with the conservation condition Eq. (7).

Inserting the form (21) into Eq. (19), the partial differential equation for $n(x, t)$ is turned into an ordinary differential equation for $H(\xi)$,

$$
H+\xi \frac{d H}{d \xi}+3 c_{b r}\left(\frac{d H}{d \xi}\right)^{2}+3 c_{b r} H \frac{d^{2} H}{d \xi^{2}}=0,
$$

or equivalently $(d / d \xi)\left(\xi H+3 c_{b r} H d H / d \xi\right)=0$. This can immediately be integrated to give (we simultaneously insert our choice $c_{b r}=2$ ) 

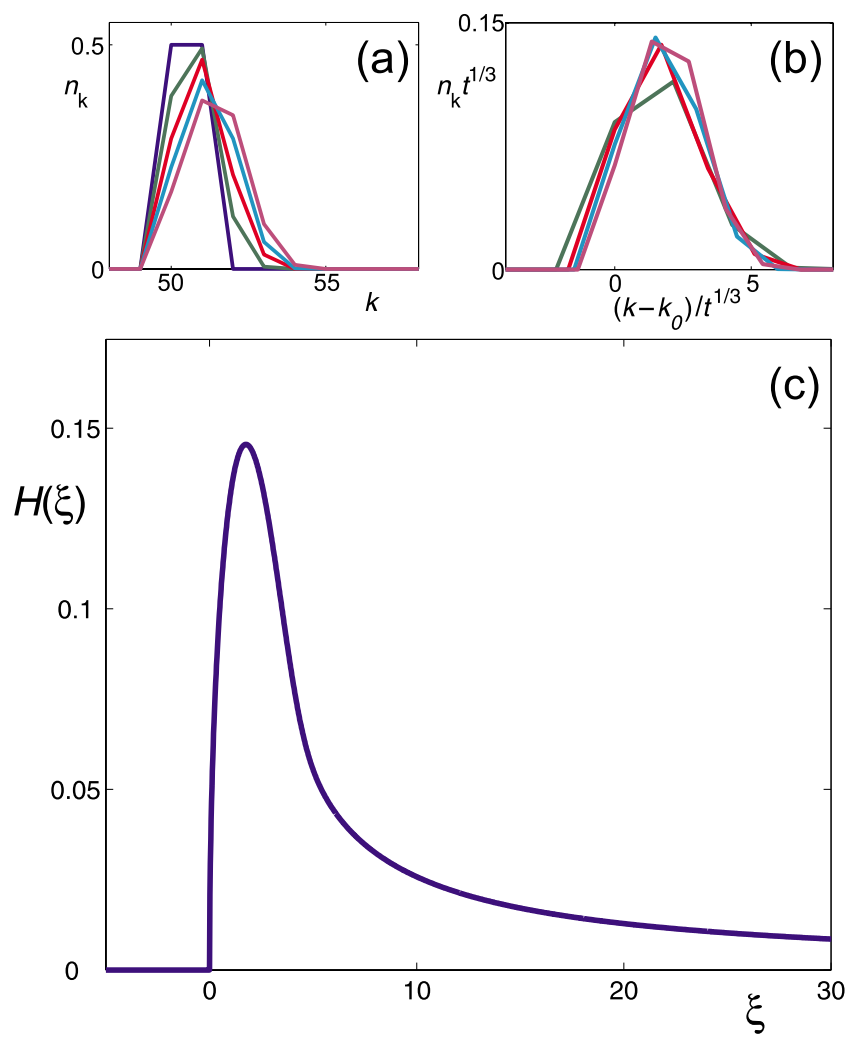

FIG. 6. (Color online) [(a) and (b)] The distributions at $t=0.1$, $0.2,0.3$, and $0.4 \mathrm{~s}$ in the numerical simulations, with $B_{L}=1000$ and $A=1 \mathrm{~m}^{-1}$ just as in Fig. 4, both (a) unscaled (where we have also included the initial condition at $t=0 \mathrm{~s}$ ) and (b) rescaled as $0.5 n_{k}(t) t^{1 / 3}$ vs $\left(k-k_{0}\right) t^{-1 / 3}$ [see Eq. (24)]. Here, $k_{0}=50$ indicates the position of the initial cluster. The cluster rapidly breaks down toward the right with the same exponent $1 / 3$ as in the symmetric diffusion stage. (c) Solution $H(\xi)$ of Eq. (23) for $\Gamma \approx 0.5$, representing the distribution for small times $t$.

$$
H\left(\xi+6 \frac{d H}{d \xi}\right)=\Gamma
$$

Here $\Gamma$ is an integration constant, the value of which is determined by initial and boundary conditions, and it is precisely at this point that the asymmetry requirement mentioned above enters the analysis.

As detailed in the Appendix, Eq. (23) has a symmetric solution (in the form of an inverted parabola) for $\Gamma=0$ and asymmetric solutions for all $\Gamma \neq 0$. The solutions for $+\Gamma$ and $-\Gamma$ are each others' mirror images with respect to the axis $\xi=0$ and the amount of asymmetry grows with increasing $|\Gamma|$.

The initial breakdown stage, with its unidirectional flow toward the right, requires a relatively large positive value of $\Gamma$. In Fig. 6(c) we give the solution $H(\xi)$ for $\Gamma=0.5$. The point $\xi=0$ represents the location of the original cluster (the boundary between the first well-filled compartment 50 and its left neighbor), so $H(0)=0$. The profile still bears the marks of the sharply peaked initial condition and the protuberance toward the right reflects the downhill stream of particles that immediately sets in for $t>0$.
How does this compare to the observations? Figure 6(a) shows how the profile evolves in the early stages from $t$ $=0.0 \mathrm{~s}$ to $t=0.4 \mathrm{~s}$, obtained directly from the flux model Eq. (6). To compare this to $H(\xi)$, we apply the self-similarity transformation Eq. (21), or rather its discrete version

$$
\begin{gathered}
n_{k}(t) \rightarrow H(\xi)=\frac{1}{c_{b r}} n_{k}(t)(A t)^{1 / 3}, \\
k-k_{0} \rightarrow \xi=\frac{k-k_{0}}{(A t)^{1 / 3}},
\end{gathered}
$$

with $A=1 \mathrm{~s}^{-1}, c_{b r}=2$, and $k_{0}=50$ (the compartment number of the initial cluster). The result is shown in Fig. 6(b). We see that the rescaled profiles indeed coincide on a curve similar to that of Fig. 6(c).

The correspondence is not perfect and should not be expected to be perfect. Not only have we neglected the firstorder terms in the above self-similarity analysis, but there is also the fact that during these very first moments, the particles move only over 3 to 4 compartments, so the flux model profiles necessarily bear conspicuous traces of the discrete compartmentalization. A strong quantitative agreement with the continuum prediction of Fig. 6(c) is therefore not feasible. Qualitatively, however, the profile according to the continuum model agrees with those in Figs. 6(a) and 6(b). More importantly, the model reproduces the correct $t^{-1 / 3}$ scaling behavior found in the rapid breakdown stage of the MD simulation [Fig. 2(c)] as well as the flux model calculations [Fig. 4(b)].

\section{B. Transient shock wave}

We now come to the second stage, the shock wave behavior, when the first-order term in the balance equation is dominant over the second-order term. This requires a sizeable $\widetilde{B}_{L}$ value (see Fig. 5 and also Sec. VI). For definiteness, we simply ignore the second-order term in Eq. (10) and assume a typical value of $\widetilde{B}_{L}=1000 \mathrm{~m}^{2}$. Since at the beginning of the shock wave stage we then have $\widetilde{B}_{L} n^{2} \approx 10$ (with $n_{\max }$ $\approx 0.10$, see Fig. 5), the flux to the left is still negligible in comparison to the flux to the right, i.e., $P(n) \approx 2 A_{1} n$.

So Eq. (10) now becomes to leading order

$$
\frac{\partial n}{\partial t}=-2 A_{1} n \frac{\partial n}{\partial x}
$$

This is known as the inviscid Burgers equation [35], famous for its traveling shock wave solutions. These solutions have the shape of a triangle [just like the waves in Figs. 2(a) and 4(a)], gradually elongating and decreasing in height

$n(x, t)= \begin{cases}\left(x-x_{1}\right) / 2 A_{1} t & \text { for } x_{1} \leq x \leq x_{1}+2\left(A_{1} t\right)^{1 / 2} \\ 0 & \text { everywhere else }\end{cases}$

The position of the front, $x_{\text {front }}(t)=x_{1}+2\left(A_{1} t\right)^{1 / 2}$, follows from the condition Eq. (7) that the area under the triangle must be equal to 1 . The height of the front is $n_{\text {front }}(x, t)$ $=\left(A_{1} t\right)^{-1 / 2}$, i.e., it decreases as the square root of time. This is the same power-law scaling (with exponent -1/2) as found in Figs. 2(c) and 4(b). 
The triangular form Eq. (26) can be understood from Eq. (25) by noting that on dimensional grounds $[33,36]$, the solution can be written in the form

$$
n(x, t)=\left(A_{1} t\right)^{-1 / 2} G(\eta),
$$

where the-dimensionless-function $G(\eta)$ depends on $x$ and $t$ only through the combined dimensionless variable $\eta=\left(x-x_{1}\right)\left(A_{1} t\right)^{-1 / 2}$. The approach is analogous to that of the previous section.

Inserting the ansatz (27) into the inviscid Burgers equation (25), the partial differential equation for $n(x, t)$ transforms into an ordinary differential equation for the function $G(\eta)$

$$
G+\eta \frac{d G}{d \eta}-4 G \frac{d G}{d \eta}=0
$$

or equivalently, $(d / d \eta)\left(\eta G-2 G^{2}\right)=0$. This equation can be integrated immediately to give $G(\eta-2 G)=C$, where the constant $C$ is determined by the boundary condition $G(0)=0$ (the shock wave is zero at the left end of the tail), i.e., $C=0$. So

$$
G(\eta-2 G)=0,
$$

and thus we find that either $G(\eta)=0$ (the trivial solution) or $G(\eta)=\frac{1}{2} \eta$.

Together, these give exactly the shock wave: the solution $G(\eta)=\frac{1}{2} \eta$ holds for $0 \leq \eta \leq \eta_{\text {front }}$ and $G(\eta)=0$ holds everywhere else. The value of $\eta_{\text {front }}$ is determined by the normalization condition $\int_{0}^{\eta_{\text {front }}} G(\eta) d \eta=1$, yielding $\eta_{\text {front }}=2$. Transforming back to the variables $x$ and $t$, via Eq. (27), the shock wave takes the form of Eq. (26).

To check the self-similarity of the shock wave, in Fig. 7(a) we perform the transformation (27) or rather its discrete version

$$
\begin{gathered}
n_{k}(t) \rightarrow G(\eta)=n_{k}(t)(A t)^{1 / 2}, \\
k-k_{1} \rightarrow \eta=\frac{k-k_{1}}{(A t)^{1 / 2}},
\end{gathered}
$$

on the profiles obtained from de MD simulations (cf. Fig. 2). Naturally, we focus on the time interval when the density falls off with an exponent close to the Burgers exponent $-1 / 2$ [see Fig. 2(c)], from 40 to 220 s. The resulting curves indeed coincide in fair approximation onto a fixed triangle.

In Fig. 7(b), the same transformation (30) is performed on the profiles from the flux model (cf. Fig. 4) in the interval $100 \mathrm{~s}<t<250 \mathrm{~s}$ where $n_{\max }(t) \propto t^{-1 / 2}$ holds approximately. Also here we find a good correspondence with the expected triangular shape.

\section{Long-time behavior: Symmetric diffusion until the boundaries of the system are reached}

Still assuming a typical value of $\widetilde{B}_{L}=1000 \mathrm{~m}^{2}$, the behavior of the flow changes when $n_{\max }$ falls below 0.01. By this time, $\widetilde{B}_{L} n^{2}$ is everywhere well below 1 , such that $\exp \left(-\widetilde{B}_{L} n^{2}\right) \approx 1-\widetilde{B}_{L} n^{2}$ and the functions $P(n)$ and $Q(n)$ [Eqs. (11) and (12)] take the form
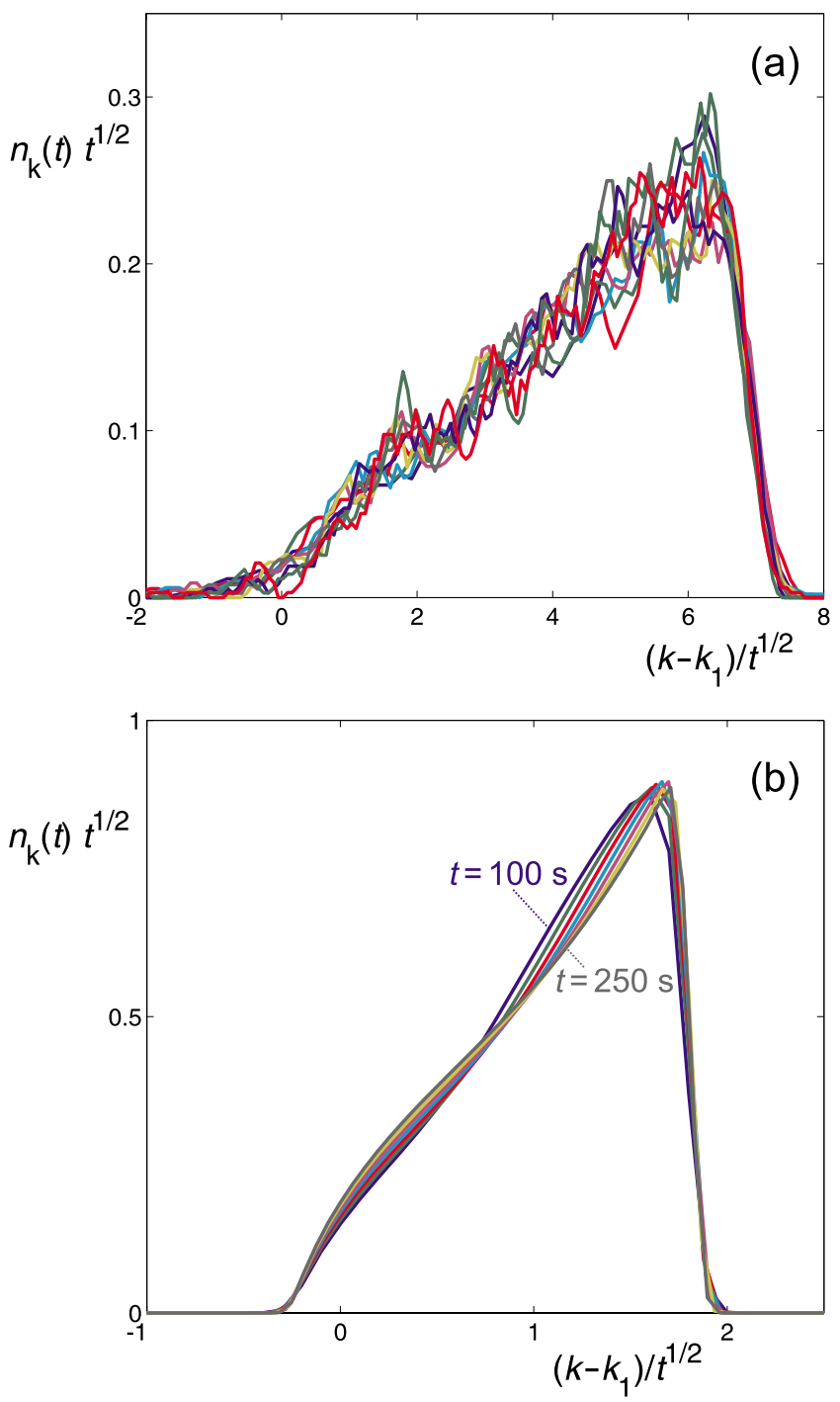

FIG. 7. (Color online) (a) The rescaled distributions during the stage when the profile has the triangular form of a Burgers shock wave in the numerical simulations, $t=40-220 \mathrm{~s}$, at time intervals of $20 \mathrm{~s}$ (i.e., at much later times than in Fig. 6). A running average over three compartments has been taken to smoothen the profiles to some extent. The density is rescaled as $n_{k}(t) t^{1 / 2}$ and the position along the staircase as $\left(k-k_{1}\right) t^{-1 / 2}$ [Eq. (30) with $A=1 \mathrm{~s}^{-1}$ ], where $k_{1}=50$ indicates the compartment in which the tail of the triangular profile is positioned, which happens to coincide with the compartment in which we placed the initial cluster. (b) Idem calculated with the flux model Eq. (6), under the same conditions as in Fig. 4, for $t=100-250 \mathrm{~s}$ at time intervals of $25 \mathrm{~s}$.

$$
P(n) \approx 4 A_{1} \widetilde{B}_{L} n^{3},
$$

$$
Q(n) \approx 2 A_{2} n\left(1-\widetilde{B}_{L} n^{2}\right)
$$

As the system is further diluted, $\widetilde{B}_{L} n^{2} \rightarrow 0$ and only $Q(n)$ $\approx 2 A_{2} n$ survives up to order $\mathcal{O}(n)$. This means that in the dilute limit (or equivalently, for $t$ sufficiently large) the balance Eq. (10) reduces to 


$$
\frac{\partial n}{\partial t}=2 A_{2} \frac{\partial}{\partial x}\left(n \frac{\partial n}{\partial x}\right) .
$$

This is, apart from the factor 2, precisely the same nonlinear heat equation as for the initial rapid breakdown-see Eq. (19) [37].

As foreseen, the term $-P(n) \partial n / \partial x$ has lost the important role it played during the shock wave regime, although as a result of it the density profile still is moving (very) slowly to the right, with a velocity proportional to $A_{1} \widetilde{B}_{L} n^{3}$ [38]. We will come back to this, but for the moment we concentrate on Eq. (32). With its full mirror symmetry with respect to left and right [noted already in the context of Eq. (19)], this equation ignores the slow drift toward the right and must therefore be interpreted as referring to the dynamics in the comoving frame.

To explain the scaling behavior observed in this third stage (i.e., the density falling off as $t^{-1 / 3}$ ), we follow the same line of reasoning as in Sec. V A and note again that on dimensional grounds, the solution to Eq. (32) can be written as [34]

$$
n(x, t)=c_{d i}\left(A_{2} t\right)^{-1 / 3} H(\xi),
$$

where the dimensionless function $H(\xi)$ depends on $x$ and $t$ only through the combined dimensionless variable $\xi=\left(x-x_{2}\right)\left(A_{2} t\right)^{-1 / 3}$. This ansatz is obviously of the same type as Eq. (21) and at once explains the observed decay exponent $1 / 3$, but this time we will set the free constant $c_{d i}$ (the subscript $d i$ stands for diffusion) equal to 1 instead of 2 . This compensates for the extra factor 2 in Eq. (32) and the resulting ordinary differential equation $H\left(\xi+6 c_{d i} d H / d \xi\right)=\Gamma$ thus becomes identical to Eq. (23) for the initial breakdown stage.

This is interesting. The same equation Eq. (23) describes the (approximate) self-similarity during the very first stages and in the long-time diffusive regime. It must be said, however, that the physics behind this equation - and in particular, the reason for the absence of the parameter $\widetilde{B}_{L}$-is quite different in each of the two cases. During the initial phase, $\widetilde{B}_{L}$ does not appear in the equation because the corresponding flux function $\widetilde{F}_{L}(n)$ is negligible compared to $\widetilde{F}_{R}(n)$ as long as $\widetilde{B}_{L} n^{2} \gg 1$. In the long-time regime, on the other hand, the absence of $\widetilde{B}_{L}$ is due to exactly the opposite feature: namely, that $\widetilde{F}_{L}(n)$ becomes equal to $\widetilde{F}_{R}(n)$ when $\widetilde{B}_{L} n^{2} \ll 1$, i.e., in the limit when the density $n$ goes to zero [37].

As noted before and as described in detail in the Appendix, Eq. (23) has a symmetric solution (an inverted parabola) for $\Gamma=0$ and asymmetric solutions for all $\Gamma \neq 0$. Now, which value of $\Gamma$ corresponds to the diffusive regime?

To answer this question, we first note that the diffusive regime does not start out with a symmetric initial condition but with the triangle-shaped profile of the Burgers shock wave (at time $t \approx 100 \mathrm{~s}$ in the $\mathrm{MD}$ simulation or $t$ $\approx 1000 \mathrm{~s}$ in the flux model calculation).

As a second step, we investigate the form of the selfsimilar profile by taking the observed density profiles from Figs. 2(a), 2(b), and 4(a) at some large values of $t$ and rescale them as follows (with $A=1 \mathrm{~s}^{-1}$ and $c_{d i}=1$ ):
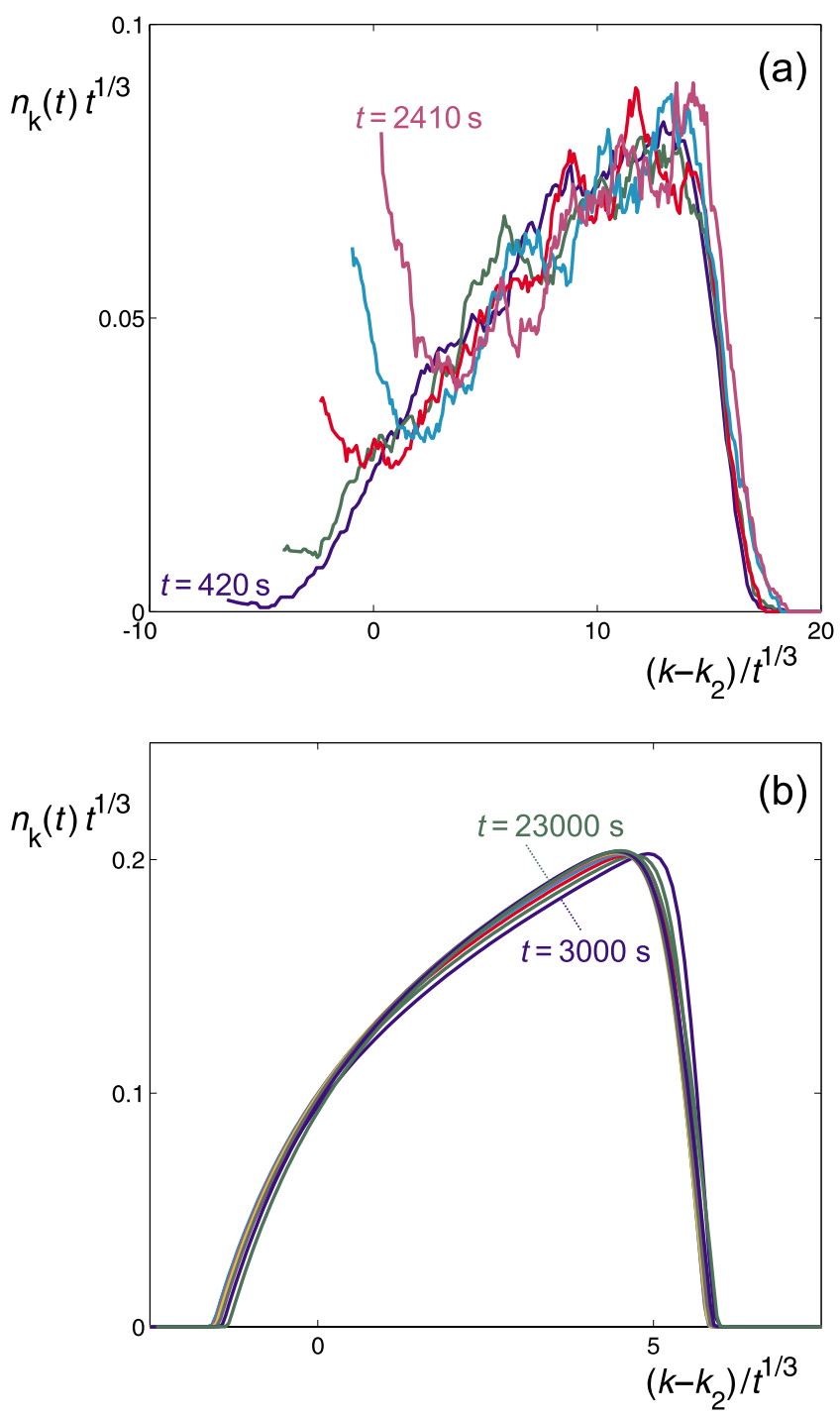

FIG. 8. (Color online) (a) The distributions at $t=420,910,1410$, 1910, and 2410 s [i.e., much later than in Fig. 7(a)] in the MD simulations, rescaled as $n_{k}(t) t^{1 / 3}$ vs $\left(k-k_{2}\right) t^{-1 / 3}$. To smoothen the profiles, a running average over 21 compartments has been taken. The fact that the best fit is obtained for $k_{2}=45$, i.e., five compartments to the left of the original cluster, reflects the transition from shock wave to diffusive regime, in which particles also move upwards. The upward twist at the left of the profiles shows that material is heaping up in the leftmost compartments. (b) Idem calculated with the flux model Eq. (6), under the same conditions as in Fig. 4, at times $t=3000-23000 \mathrm{~s}$ at time intervals of $2500 \mathrm{~s}$, and rescaled as $n_{k}(t) t^{1 / 3}$ vs $\left(k-k_{2}\right) t^{-1 / 3}$ with $k_{2}=43$. Although the diffusion is governed by the same Eq. (23) as the initial breakdown, the profiles differ considerably since the two regimes start out from very different situations (cf. Fig. 6).

$$
\begin{gathered}
n_{k}(t) \rightarrow H(\xi)=\frac{1}{c_{d i}} n_{k}(t)(A t)^{1 / 3}, \\
k-k_{2} \rightarrow \xi=\frac{k-k_{2}}{(A t)^{1 / 3}} .
\end{gathered}
$$

The result is shown in Fig. 8(a) and 8(b) for the MD simu- 
lations and the flux model, respectively; we have used values for $k_{2}$ that are slightly lower than the initial position of the cluster. In both cases the rescaled profiles coincide on a curve in which one recognizes an evolved form of the triangular shock wave, spread out in both directions and with all its sharp features being softened. The upward twist at the left-hand side of Fig. 8(a) indicates that material is heaping up in the leftmost compartments, where it meets the boundary of the system and is unable to diffuse further to the left.

All the above evidence seems to point to some relatively large negative value of the integration constant $\Gamma$. In Fig. 9(a), we show the numerical solution $H(\xi)$ of Eq. (23) for $\Gamma \approx-0.5$ and indeed, at first glance, the shape seems to resemble the curves in Fig. 8. If one is willing to translate the numerical profiles of Fig. 8(a) toward the left [see the gray curves in Fig. 9(a)] the coincidence becomes even striking. However, this translation is physically not justified. The profile $H(\xi)$ for $\Gamma=-0.5$ is almost entirely positioned to the left of $\xi=0$, corresponding to a situation in which the particles would have moved exclusively upstream, whereas in reality [both in the MD simulations of Fig. 8(a) as in the flux model calculation of Fig. 8(b)], the particles have preferentially moved downstream.

The true self-similarity of the diffusive process is only revealed if we go to really long times. We therefore performed flux model calculations in a much larger system of $K=10000$ compartments-with all particles initially distributed equally over compartments 4000 and 4001—and over a much longer time span than before [39]. The results of these calculations are shown in Fig. 9(b), where we see the profiles at the exponentially increasing time scales $t=10^{3}, 10^{5}, 10^{7}$, and $10^{9} \mathrm{~s}$. Here, the real long-time behavior becomes visible. The profile, extremely diluted by now, slowly converges toward the symmetric solution of Eq. (23), i.e., the one with $\Gamma=0$ represented by the dotted inverted parabola. [For better comparison, the support of each profile in Fig. 9(b) has been rescaled to coincide with the support of $H(\xi)$ with $\Gamma=0$.] It takes exceedingly long before the symmetric diffusion process described by Eq. (32) has finally repaired the asymmetries in the profile acquired during the previous two stages, but it keeps working at it, since the symmetric profile is the only sensible self-similar solution to Eq. (32). It is merely thanks to the extraordinary slowness of the convergence that the profiles for a limited time span [such as those in Figs. $8(\mathrm{a})$ and $8(\mathrm{~b})$ ] can appear to be self-similar with a nonzero value of $\Gamma$.

In this same context one may also note that the exponent $1 / 3$ corresponds to a slow kind of diffusion. Normal random walker diffusion has an exponent $1 / 2$, i.e., the height of the profile decays as $t^{-1 / 2}$ and its width grows as $t^{1 / 2}$, which is quicker than in the present case. The reason for the slowness of the diffusion in our system is the nonlinearity in Eq. (32) [the extra $n$ in the right-hand term as compared to the linear heat equation $\left.\partial n / \partial t=D \partial^{2} n / \partial x^{2}\right]$. This slows down the dynamics where $n$ is small, i.e., precisely at the outer edges of the profile, hence the width grows slower than $t^{1 / 2}$ [26].

For any finite number of compartments $K$, the diffusion alone would eventually lead to a constant level $n_{k}=1 / K$ along the entire length of the staircase. However, in reality, there will remain a small bias toward the right [correspond-
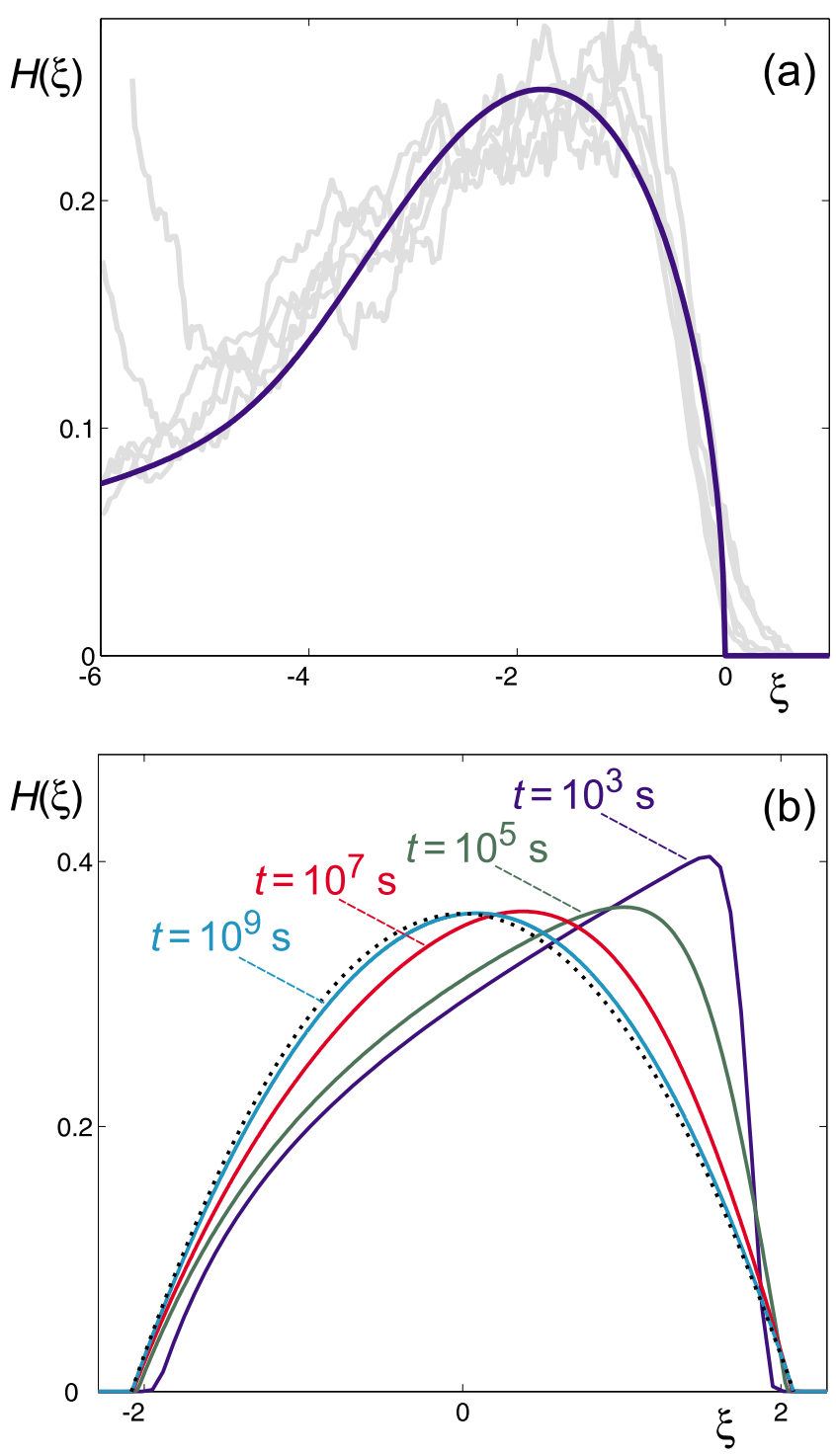

FIG. 9. (Color online) (a) Apparent but untrue self-similarity. Solution $H(\xi)$ of Eq. (23) for $\Gamma=-0.5$. Although the shape bears a marked resemblance to that of the MD results of Fig. 8(a) (gray shaded curves), the depicted coincidence has only been obtained via a nonadmissible translation of the MD profiles to the left. (b) Nonconspicuous but true self-similarity. Rescaled flux model profiles obtained in a much larger system (10 000 compartments with the cluster initially in compartment 4000 and its neighbor) and on a much longer time scale than in Fig. 4, namely, at $t=10^{3}, 10^{5}, 10^{7}$, and $10^{9} \mathrm{~s}$. The values of $B_{L}=1000$ and $A=1 \mathrm{~s}^{-1}$ are unaltered. These profiles reveal a slow convergence toward the symmetric solution of Eq. (23), i.e., the one with $\Gamma=0$ (see also the Appendix).

ing to the term $-P(n) \partial n / \partial x$, see our discussion below Eq. (32)] because $F_{R}\left(n_{k}\right)$ is slightly larger than $F_{L}\left(n_{k}\right)$ even for the smallest, but necessarily nonzero, values of $n_{k}$. Among other things, this means that the heaping effect at the lefthand boundary seen in Fig. 8(a) is really a transient phenomenon, which in the course of time will flatten out again. The final state for $t \rightarrow \infty$ is characterized by a flux balance between adjacent compartments, $F_{R}\left(n_{k-1}\right)=F_{L}\left(n_{k}\right)$ for all $k$ $=2, \ldots, K$, or equivalently $n_{k-1}^{2}=n_{k}^{2} \exp \left(-B_{L} n_{k}^{2}\right)$, which in the dilute limit $B_{L} n_{k}^{2} \ll 1$ reduces to 

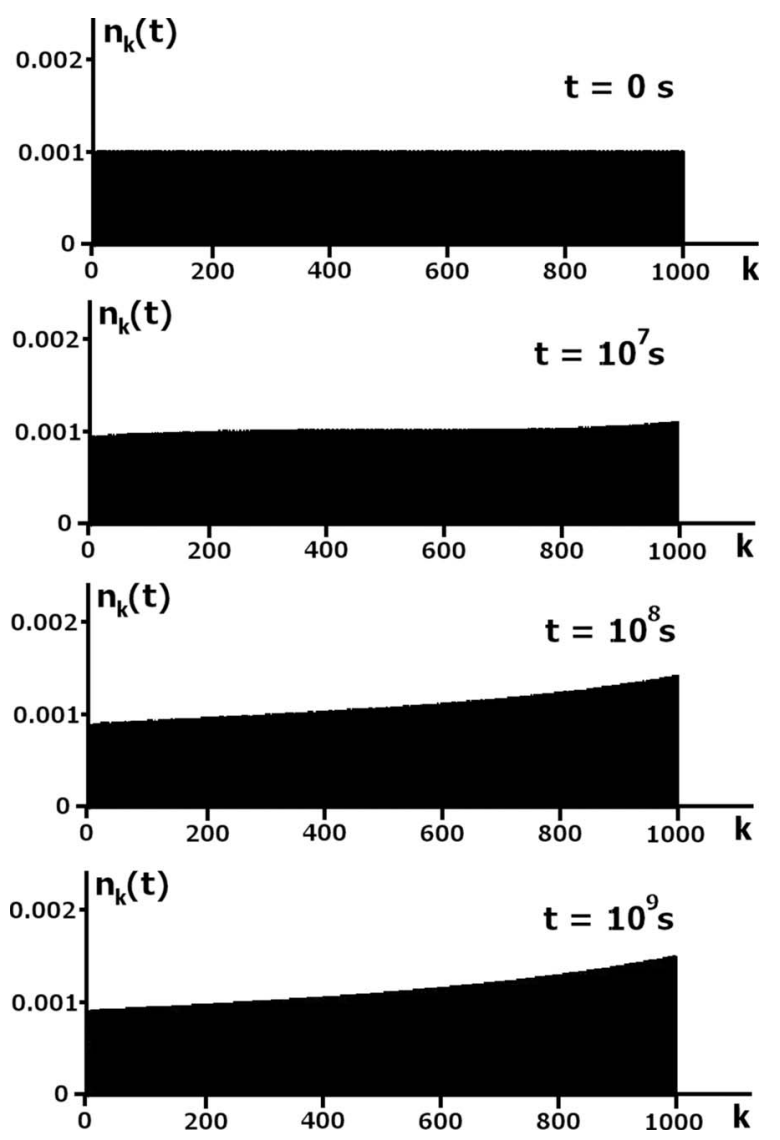

FIG. 10. Approach toward the final state on a staircase of finite length. Starting out from a dilute homogeneous distribution over a staircase with $K=1000$ steps (and shaking parameters $B_{L}=1000$, $A=1 \mathrm{~s}^{-1}$ ), we witness how the system slowly evolves toward a biased distribution in which the fluxes between all neighboring compartments are in equilibrium. In the last snapshot $\left(t=10^{9} \mathrm{~s}\right)$, the convergence is complete, i.e., the density profile will not visibly change anymore. This profile is accurately described by Eq. (36) with $C=1.5610^{6}$.

$$
n_{k-1}=n_{k}\left[1-\frac{1}{2} B_{L} n_{k}^{2}+O\left(B_{L} n_{k}^{2}\right)^{2}\right] .
$$

This demonstrates that $n_{k-1}$ is indeed a bit smaller than $n_{k}$. The approach to the final state is illustrated in Fig. 10, which starts out from a dilute homogeneous distribution on a staircase consisting of $K=1000$ compartments. On the time scale of this figure, we see how the system settles into the biased equilibrium state described by Eq. (35).

In fact, this final distribution can be evaluated analytically. Given a sufficiently long staircase, Eq. (35) may be rewritten in the differential form $n_{k}-n_{k-1}=d n_{k} / d k=\frac{1}{2} B_{L} n_{k}^{3}$, which is readily solved to give

$$
n_{k}=\frac{1}{\sqrt{C-B_{L} k}} \text { for } k=1, \ldots, K .
$$

The integration constant $C$ follows from the conservation condition $\int_{1}^{K} n_{k} d k=\left(2 / B_{L}\right)\left(\sqrt{C-B_{L}}-\sqrt{C-B_{L} K}\right)=1$. For $B_{L}$ $=1000$ and $K=1000$, we find $C=1561001$, yielding a theoretical density profile Eq. (36) that perfectly matches the curve in the last snapshot of Fig. 10.
It is good to stress that the emergence of this profile is a finite-size effect. On an infinitely long, unbounded staircase, the diffusion would continue forever and the density profile would simply be a hill [as in Fig. 9(b)] decaying as $t^{-1 / 3}$ with a decreasing bias toward the lower compartments.

\section{VARYING THE SHAKING PARAMETER $\widetilde{B}_{L}$}

The relative duration of the three stages - and the clarity with which they show up-depends on the value of $\widetilde{B}_{L}$. This can be seen from the ratio Eq. (18), which [via the expressions for $P(n)$ and $Q(n)]$ is a function of $\widetilde{B}_{L} n^{2}$. Also, the final equilibrium state (for any staircase of finite length) depends on the value of $\widetilde{B}_{L}$, as can be seen directly from Eq. (35). The bias toward the lower compartments becomes more pronounced for growing $\widetilde{B}_{L}$.

In this section we discuss the effects of varying the value of $\widetilde{B}_{L}$. Up to now, we have concentrated on the case $\widetilde{B}_{L}$ $=1000 \mathrm{~m}^{2}$, corresponding to our MD simulations, but what happens when we make $\widetilde{B}_{L}$ smaller or larger? Such a change is easily accomplished by increasing (respectively, decreasing) the amplitude and frequency of the shaking or by changing any of the other quantities appearing in the expression (3) for $B_{L}=\widetilde{B}_{L} /(\Delta x)^{2}, \Delta x$ being the width of a compartment.

In the limits of small and large $\widetilde{B}_{L} n^{2}$, the ratio Eq. (18) takes the form

$$
\begin{aligned}
& R(n) \approx \frac{2 \widetilde{B}_{L} n}{\gamma^{\prime}} \quad \text { if } \widetilde{B}_{L} n^{2} \ll 1 \\
& R(n) \approx \frac{2}{\gamma^{\prime} n} \quad \text { if } \widetilde{B}_{L} n^{2} \gg 1 .
\end{aligned}
$$

The two crossover points in Fig. 5, corresponding to $R(n)$ $=1$, can now be approximated using the above equation. The crossover from the initial rapid breakdown to the shock wave happens at a relatively large value $n=n_{\mathrm{i} \rightarrow \mathrm{i}}$, and hence we may expect the second approximation of Eq. (37) to hold, i.e., $2 / \gamma^{\prime} n_{\mathrm{i} \rightarrow \mathrm{ii}} \approx 1$. On the other hand, the crossover from the shock wave to the diffusive behavior happens at a much smaller value $n=n_{\mathrm{ii} \rightarrow \mathrm{iii}}$, so now we can invoke the first approximation of Eq. (37). This yields [40]

$$
n_{\mathrm{i} \rightarrow \mathrm{ii}} \approx \frac{2}{\gamma^{\prime}} \quad \text { and } \quad n_{\mathrm{ii} \rightarrow \mathrm{iii}} \approx \frac{\gamma^{\prime}}{2 \widetilde{B}_{L}},
$$

leading to the following important conclusions:

First, the crossover from the rapid breakdown (i) to the shock wave (ii) is independent of $\widetilde{B}_{L}$ and thus cannot be influenced by adjusting the amplitude or frequency of the driving. This has already been illustrated in Fig. 5, where we saw that this crossover happens at exactly the same value of $n_{\max }$ both for $\widetilde{B}_{L}=1000 \mathrm{~m}^{2}$ and $\widetilde{B}_{L}=10000 \mathrm{~m}^{2}$.

Second, the crossover from the shock wave (ii) to the diffusive behavior (iii) is inversely proportional to $\widetilde{B}_{L}$. Thus, if we increase $\widetilde{B}_{L}$ (e.g., by decreasing the shaking frequency), 


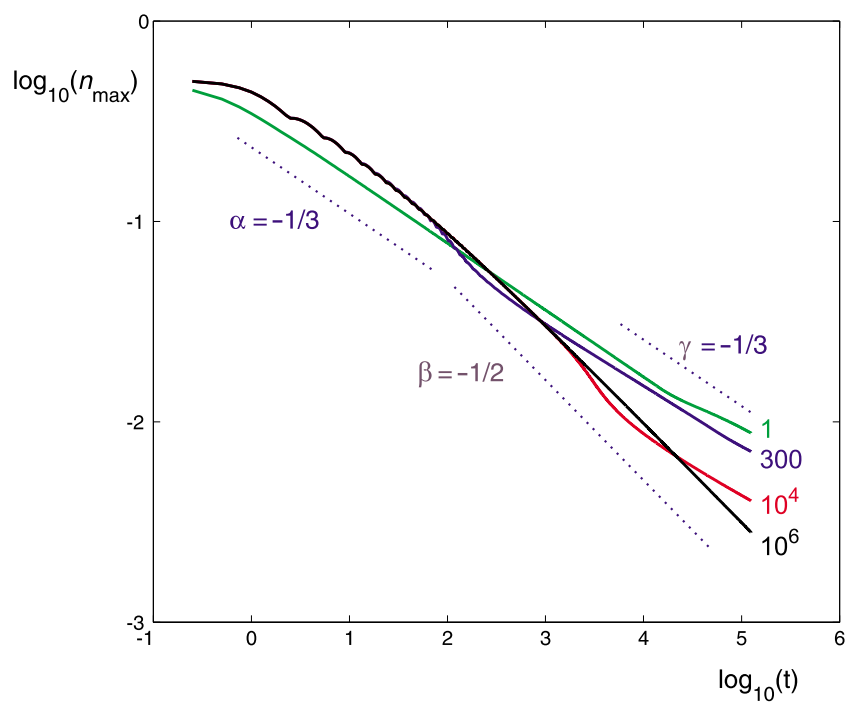

FIG. 11. (Color online) Decay of $n_{\max }(t)$ calculated from the flux model Eq. (6) for four different values of $B_{L}$, each one starting from the same initial condition $n_{50}(0)=n_{51}(0)=0.50$ in a system of $K=1000$ compartments. (a) For $B_{L}=1$ (green curve), the breakdown of the cluster directly goes over into symmetric diffusion, without intervention of any shock wave, hence the slope is permanently close to $-1 / 3$. The deviation beyond $\log _{10} t=4.3$ is caused by the left boundary, which is reached relatively soon at this strong shaking. [(b) and (c)] For $B_{L}=300$ and $10^{4}$ (blue and red curves) all three regimes are distinguishable [(i) initial breakdown with slope $-1 / 3$; (ii) shock wave with slope $-1 / 2$; (iii) diffusion with slope $-1 / 3]$, with the length of the shock wave regime growing for increasing $B_{L}$. (d) For $B_{L}=10^{6}$ (black curve), the shock wave regime extends all the way to the end of the depicted time interval, as the crossover takes place at a value $n_{\mathrm{ii} \rightarrow \mathrm{iii}}<10^{-3}$, in agreement with Eq. (38). Dashed lines with slopes $-1 / 3,-1 / 2$, and (again) $-1 / 3$ have been added for reference.

the crossover value $n_{\mathrm{ii} \rightarrow \mathrm{iii}}$ becomes smaller and consequently the duration of the shock wave stage increases (see again Fig. 5). Vice versa, below $\widetilde{B}_{L} \approx 100 \mathrm{~m}^{2}$, we find that $\log _{10} R(n)$ always remains negative [i.e., $R(n)<1$ ] and hence the shock wave stage vanishes altogether. That is, for small values of $\widetilde{B}_{L}$, or strong shaking, the initial breakdown goes over directly into the diffusive regime. The physical reason for this is that, at strong shaking, the height difference between the left and right apertures is simply not sufficient to generate the considerable flux unbalance required for a shock wave. (Not surprisingly, in this case, also the bias in the final state is very small.)

These conclusions are confirmed by Fig. 11 where we plot the maximum profile height $n_{\max }(t)$, calculated from the discrete flux model, for four different values of $B_{L}$ :

(1) For $B_{L}=1$ (very strong shaking), we only retrieve the $t^{-1 / 3}$ decay, meaning there is no shock wave in this case, just as expected.

(2) and (3) For $B_{L}=300$ and $B_{L}=10000$, we find all three stages. As anticipated on the basis of Eq. (38), the transition from the initial breakdown (slope $-1 / 3$ ) to the shock wave (slope -1/2) occurs simultaneously for both $B_{L}$ valuesaround $\log _{10}=1.6$ or $t \approx 40 \mathrm{~s}$-whereas the next transition to the diffusive stage (slope $-1 / 3$ again) occurs much earlier for the small $B_{L}$ value than for the larger one. We also note that this second transition is accompanied by a momentary steepening of the slope well beyond the Burgers exponent of $-1 / 2$. This means that the decay of $n_{\max }(t)$ is momentarily accelerated, which has to do with the fact that the position of the maximum is now being transferred from the front (where it was located during the shock wave regime) toward the center of the profile.

(4) Also for $B_{L}=10^{6}$ (very weak shaking), one gets all three stages, but the transition to the third, diffusive stage takes place beyond the end of the time interval shown in Fig. 11 . We therefore see a clear $t^{-1 / 2}$ scaling behavior all the way up to the right-hand side of the figure. Indeed, the shock wave regime can be protracted for arbitrarily long times by going to higher and higher values of $B_{L}$. All in all, the value $B_{L}=1000$ (or, for the continuum version, $\widetilde{B}_{L}=1000 \mathrm{~m}^{2}$ ) adopted in the main part of the paper is seen to be an adequate choice for bringing out the full dynamical potential of the system.

\section{CONCLUSION}

In conclusion, we have shown that a pile of granular material, when it is brought into motion on a vertically vibrating staircase, goes through three stages: (i) first, there is a rapid breakdown of the cluster, with its particles moving freely downstream. (ii) Soon afterwards, the downward flow organizes itself in the form of a Burgers shock wave and (iii) when the granular material is sufficiently diluted, the flow becomes diffusive with an anomalous diffusion exponent $-1 / 3$. In this third stage, a substantial part of the particles is moving upstream toward the top of the staircase.

The observed power-law decay of $n_{\max }(t)$ (the maximum particle density at time $t$ ) has been explained in terms of a dynamical flux model, in particular by the partial differential equation constituting the continuum version of this model. We identified the appropriate limiting cases of this equation associated with the three successive stages and determined the corresponding self-similarity solutions. This yielded the power laws (i) $n_{\max }(t) \propto t^{-1 / 3}$, (ii) $n_{\max }(t) \propto t^{-1 / 2}$, and (iii) $n_{\max }(t) \propto t^{-1 / 3}$, in full agreement with the observations.

Let us recapitulate the main points of the paper by considering the situation of Fig. 12, where we start out with one large and one small cluster [41]. As soon as the shaking is turned on, the granular material starts to move downward and after a fast initial breakdown, we see the spontaneous formation of two shock waves (see the snapshot at $t=80 \mathrm{~s}$ ). As indicated by the arrows, the velocity of the large wave is considerably larger than that of the smaller one; this can be understood from $v_{\text {shock }}=d x_{\text {front }} / d t=\left(A_{1} / t\right)^{1 / 2}=A_{1} n_{\text {front }}$ [cf. Eq. (26)]. As a result, the denser shock wave overtakes the more diluted one, momentarily gaining in density-and hence velocity - as it does so. The snapshot at $t=170 \mathrm{~s}$ illustrates this. Afterwards, as the height of the profile diminishes, we witness the crossover to a diffusive flow in which the profile gradually takes on a more symmetric shape (see the snapshots at $t=1300 \mathrm{~s}$ and $t=5000 \mathrm{~s}$ ). The extent of the upstream motion- toward the left-can be read off from the snapshot at $t=5000 \mathrm{~s}$, where about $4 \%$ of the granular ma- 

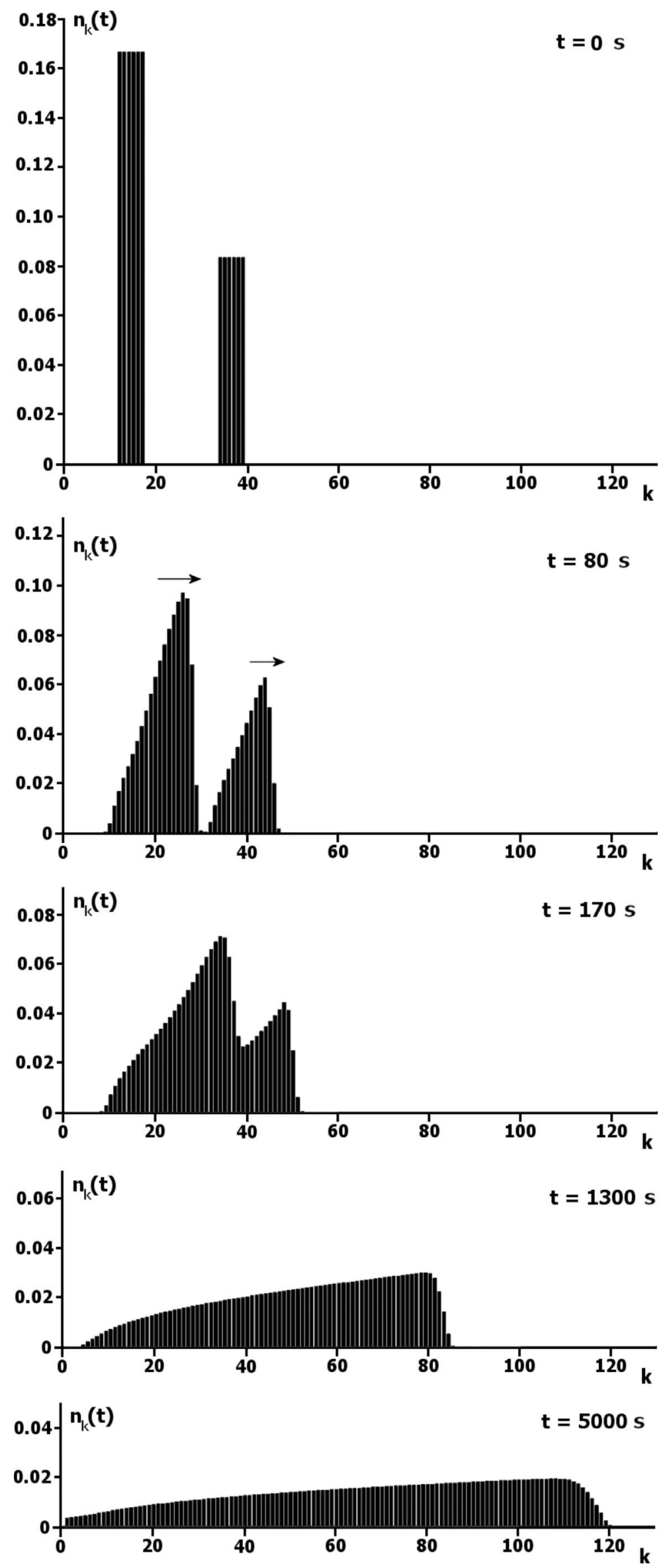

FIG. 12. Starting out with a large heap on the steps $k=12, \ldots, 17$ and a small one at $k$ $=34, \ldots, 39$ (see the snapshot at $t=0 \mathrm{~s})$, one witnesses first a rapid breakdown and formation of two Burgers-like shock waves $(t=80 \mathrm{~s})$. The higher wave travels faster and overtakes the smaller one $(t=170 \mathrm{~s})$, leading to a single density profile that diffuses out into both directions $(t=1300 \mathrm{~s})$. Eventually, the upstream flow toward the left becomes almost equal to the flow toward the right. At $t=5000 \mathrm{~s}$, the compartments 1-11 have already received a substantial amount of material. The shaking parameters are $B_{L}$ $=2250$ [41] and $A=1 \mathrm{~s}^{-1}$, which makes the dynamics of the large cluster directly comparable to Figs. 3-5, 6(a), 6(b), 7(b), 8(b), and 9(b). The velocity of the large shock wave at $t=80 \mathrm{~s}$ is 0.100 compartments/s, against 0.077 compartments/s for the smaller one (indicated by the arrows). 
terial is located on the first 11 steps of staircase, i.e., further toward the left than any of the material in the initial situation. Given that the staircase has a finite length, in the longtime limit $t \rightarrow \infty$ the system will attain the diluted equilibrium state (with a bias toward the right, cf. Fig. 10) characterized by a balance of fluxes between every adjacent pair of compartments.

\section{ACKNOWLEDGMENTS}

We wish to thank Tassos Bountis, Yannis Dimakopoulos, Detlef Lohse, and Dimitris Tsoubelis for stimulating discussions. Thanks are also due to Marcel Kloosterman for performing accompanying experiments on a 25-compartment staircase [22]. This work is sponsored by the Carathéodory Programme of the University of Patras under Grant No. C167.

\section{APPENDIX: SOLVING EQ. (23), CONCERNING THE SELF-SIMILARITY DURING THE INITIAL BREAKDOWN AND THE LATER DIFFUSIVE STAGE}

In this appendix, we solve and explore some properties of Eq. (23), together with the particle conservation condition Eq. (7) written in terms of $H$ and $\xi$,

$$
\begin{gathered}
H\left(\xi+6 \frac{d H}{d \xi}\right)=\Gamma \\
\int_{-\infty}^{\infty} H(\xi) d \xi=1 .
\end{gathered}
$$

We first turn to the case $\Gamma=0$. The corresponding equation $H(\xi+6 d H / d \xi)=0$ is easily solved to give a combination of $H(\xi)=0$ (the trivial solution) and $H(\xi)=H(0)-\xi^{2} / 12$. Together, these two solutions comprise a symmetrically decaying profile, in the form of an inverted parabola between $\xi_{ \pm}$ $= \pm \sqrt{12 H(0)}= \pm 2.08$, and zero everywhere else [26]. The value of $H(0)$ is determined by the normalization $\int_{\xi}^{\xi+} H(\xi) d \xi=1$, yielding $H(0)=3^{1 / 3} / 4 \approx 0.361$. A plot of this symmetric solution is shown in Fig. 13 (dashed black line).

Solving Eqs. (A1) and (A2) for $\Gamma \neq 0$ presents more difficulties and it is convenient to first present some of its properties:

(a) The solutions for $\Gamma$ and $-\Gamma$ are each other's mirror image with respect to the axis $\xi=0$. This can be seen by applying the transformation $\xi \rightarrow \xi^{\prime}=-\xi$ to Eq. (A1), which gives the identical equation with $-\Gamma$ instead of $\Gamma$. Hence it is sufficient to study the case $\Gamma>0$.

(b) The dependence of $\Gamma$ can be transferred to the particle conservation condition by a simple rescaling: $\widetilde{H} \equiv \Gamma^{-2 / 3} H, \widetilde{\xi}$ $\equiv \Gamma^{-1 / 3} \xi$, which transforms Eqs. (A1) and (A2) into

$$
\tilde{H}\left(\widetilde{\xi}+6 \frac{d \tilde{H}}{d \widetilde{\xi}}\right)=1,
$$

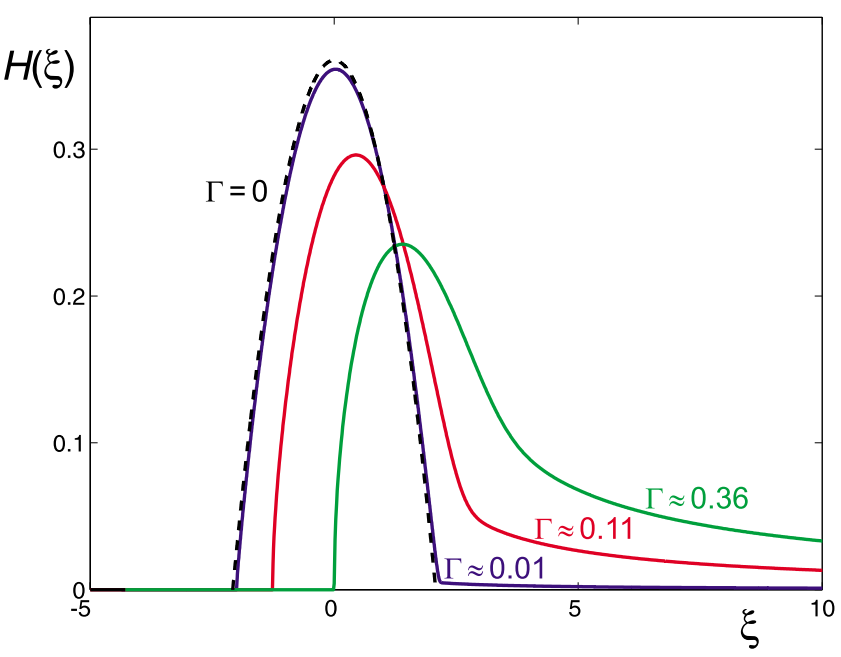

FIG. 13. (Color online) Solutions of Eq. (A1), or Eq. (23) in the main text, normalized on the interval $-10 \leq \xi \leq 10$. Dashed black curve is the symmetric case for $\Gamma=0$, which can be solved analytically. Solid blue, red, and black curves that deviate increasingly from the symmetric solution correspond to $\Gamma \approx 0.01, \Gamma \approx 0.11$, and $\Gamma \approx 0.36$, respectively. These were determined numerically. Negative values of $\Gamma$ yield solutions that are the mirror images (with respect to $\xi=0$ ) of those for positive $\Gamma$.

$$
\int_{-\infty}^{\infty} \tilde{H}(\widetilde{\xi}) d \widetilde{\xi}=1 / \Gamma .
$$

This provides us with a welcome alternative to the somewhat laborious task of numerically shooting-for some fixed $\Gamma$ - the solution of Eq. (A1) that simultaneously satisfies the conservation condition Eq. (A2). We can simply generate any solution to Eq. (A3) [e.g., by fixing $\widetilde{H}(0)$ to some constant value and numerically solving the differential equation forward and backward in $\tilde{\xi}]$, determine $\Gamma$ by integrating this solution via Eq. (A4), and finally transform back to $H(\xi)$.

(c) For the maximum $H_{\max }$ at $\xi=\xi_{\max }$, we have $H_{\max } \xi_{\max }$ $=\Gamma$. This is because in the maximum, the slope is zero, $d H /\left.d \xi\right|_{\xi_{\max }}=0$, and evaluating Eq. (A1) in $\xi=\xi_{\max }$ then gives the quoted result.

(d) Similarly, we have $6 H(0) d H / d \xi(0)=\Gamma$, which follows from inserting $\xi=0$ into Eq. (A1).

(e) For all $\Gamma \neq 0, H(\xi)$ goes asymptotically to zero as $H(\xi) \propto \xi^{-1}$ for $\xi \rightarrow \infty$. This can be seen by writing Eq. (A1) in the form $H(\xi)=\Gamma /(\xi+6 d H / d \xi)$. Now if $H(\xi)$ goes to zero in the limit $\xi \rightarrow \infty$, then certainly $d H / d \xi$ will and thus $H(\xi)$ $\approx \Gamma / \xi$.

An important consequence of the last property is that the integral of $H(\xi)$ diverges when $\xi$ goes toward infinity and therefore the particle conservation condition cannot be imposed onto the infinite domain $[-\infty, \infty]$. A practical workaround is to restrict the upper integration boundary to some finite value $\xi_{\text {sup }}$ (if $\Gamma>0$ ) or the lower integration boundary to $\xi_{\text {inf }}($ if $\Gamma<0$ ), corresponding, e.g., to the size of the system. This however limits the interpretation of such a solution as a self-similar profile, since it would imply that the 
physical boundary shifts in time. From the definition of $\xi$ [Eq. (33)] we have $x_{\text {sup }}-x_{2}=\left(A_{2} t\right)^{1 / 3} \xi_{\text {sup. Any solution with }}$ $\Gamma \neq 0$ can therefore only be an approximation to the profile for some finite time interval, just as we concluded in the main text.

In Fig. 13, we present (apart from the analytic symmetric solution for $\Gamma=0$ ) three numerical solutions to Eqs. (A1) and (A2) with $\xi_{\text {sup }}=10$ for increasing positive values of $\Gamma$, namely, $\Gamma \approx 0.01, \Gamma \approx 0.11$, and $\Gamma \approx 0.36$. The solution with $\Gamma \approx 0.36$ corresponds to the most asymmetric solution that still crosses the point $\xi=0$ at some finite height. Increasing $\Gamma$ further produces profiles that lie entirely to the right of $\xi$ $=0$. This is precisely what fits the observations during the initial phase, when the particle flow is unidirectional toward the right [see the solution for $\Gamma=0.5$ in Fig. 6(c)]. On the other hand, such a one-sided solution (or its mirror image for negative $\Gamma$ ) does not agree with the observed density profiles during the diffusive regime. Indeed, it was upon these grounds that the solution for $\Gamma=-0.5$ in Fig. 9(a) was disqualified.
[1] S. B. Savage and K. Hutter, J. Fluid Mech. 199, 177 (1989).

[2] G. Metcalfe, T. Shinbrot, J. J. McCarthy, and J. M. Ottino, Nature (London) 374, 39 (1995).

[3] J. M. N. T. Gray and K. Hutter, Phys. Blatt. 54, 37 (1998).

[4] A. Daerr and S. Douady, Nature (London) 399, 241 (1999).

[5] O. Pouliquen, Phys. Fluids 11, 1156 (1999).

[6] Y.-C. Tai, K. Hutter, and J. M. N. T. Gray, Dense Granular Avalanches: Mathematical Description and Experimental Validation, Lecture Notes in Physics 582 (Springer-Verlag, Berlin, 2001).

[7] I. S. Aranson and L. S. Tsimring, Phys. Rev. E 64, 020301(R) (2001).

[8] J. Rajchenbach, Phys. Rev. Lett. 89, 074301 (2002).

[9] L. Bocquet, J. Errami, and T. C. Lubensky, Phys. Rev. Lett. 89, 184301 (2002).

[10] S. P. Pudasaini and K. Hutter, Avalanche Dynamics: Dynamics of Rapid Flows of Dense Granular Avalanches (SpringerVerlag, Berlin, 2006).

[11] E. Martinez, C. Pérez-Penichet, O. Sotolongo-Costa, O. Ramos, K. J. Måløj, S. Douady, and E. Altshuler, Phys. Rev. E 75, 031303 (2007).

[12] Y. Forterre and O. Pouliquen, Annu. Rev. Fluid Mech. 40, 1 (2008).

[13] T. M. Knowlton, J. W. Carson, G. E. Klinzing, and W.-C. Yang, Chem. Eng. Prog. 90, 44 (1994).

[14] H. Jaeger, S. Nagel, and R. Behringer, Rev. Mod. Phys. 68, 1259 (1996).

[15] J. Duran, Sand, Powders, and Grains: An Introduction to the Physics of Granular Materials (Springer, New York, 2000).

[16] D. Chowdhury, L. Santen, and A. Schadschneider, Phys. Rep. 329, 199 (2000).

[17] D. Helbing, Rev. Mod. Phys. 73, 1067 (2001).

[18] K. van der Weele, W. Spit, T. Mekkes, and D. van der Meer, in Traffic and Granular Flow '03, edited by S. Hoogendoorn, S. Luding, and D. E. Wolf (Springer, Berlin, 2005), pp. 569-578.

[19] M. C. Cross and P. C. Hohenberg, Rev. Mod. Phys. 65, 851 (1993).

[20] I. Goldhirsch, Annu. Rev. Fluid Mech. 35, 267 (2003).

[21] I. S. Aranson and L. S. Tsimring, Rev. Mod. Phys. 78, 641 (2006).

[22] The reason we take so many compartments $(K=1000)$ is to give the flow enough space to develop freely, without being hindered by boundary effects. In a demonstration experiment on a short staircase of $K=25$ steps, we witnessed only the first two stages: the fast breakdown of the cluster and the formation of a shock wave. Due to the limited number of compartments, the shock wave reached the end of the system before it was sufficiently diluted to give way to the symmetric diffusion. The particles heaped up in the 25th compartment, at the lower end of the staircase, and henceforth $n_{\max }$ simply remained too large to prompt any appreciable upstream motion.

[23] J. Eggers, Phys. Rev. Lett. 83, 5322 (1999).

[24] K. van der Weele, D. van der Meer, M. Versluis, and D. Lohse, Europhys. Lett. 53, 328 (2001).

[25] D. van der Meer, K. van der Weele, and D. Lohse, Phys. Rev. E 63, 061304 (2001).

[26] D. van der Meer, K. van der Weele, and D. Lohse, Phys. Rev. Lett. 88, 174302 (2002).

[27] K. van der Weele, R. Mikkelsen, D. van der Meer, and D. Lohse, in The Physics of Granular Matter, edited by H. Hinrichsen and D. E. Wolf (Wiley-VCH, Weinheim, Germany, 2004), pp. 117-139.

[28] D. van der Meer, K. van der Weele, P. Reimann, and D. Lohse, J. Stat. Mech.: Theory Exp. (2007) P07021.

[29] K. van der Weele, Contemp. Phys. 49, 157 (2008).

[30] R. Mikkelsen, K. van der Weele, D. van der Meer, M. van Hecke, and D. Lohse, Phys. Rev. E 71, 041302 (2005).

[31] Alternatively, one may interpret this convention as introducing a length scale in which the unit of measurement is given by the compartment width $\Delta x$ and read "compartment width" wherever the unit "meter" is used.

[32] A. D. Polyanin and V. F. Zaitsev, Handbook of Nonlinear Partial Differential Equations (Chapmann and Hall/CRC, Boca Raton, 2004).

[33] G. J. Barenblatt, Scaling, Self-Similarity, and Intermediate Asymptotics (Cambridge University Press, Cambridge, England, 1996).

[34] The solution of Eq. (19) under the conservation condition Eq. (7) is completely determined by the four dimensional quantities $x-x_{0}, t, n$, and $A_{2}$. By Buckingham's $\Pi$ theorem, it follows [33] that there exists a description in terms of two dimensionless quantities. The position $x-x_{0}$ and time $t$ (together with $A_{2}$, whose dimension is $\left.L^{3} / T\right)$ combine into the dimensionless parameter $\xi=\left(x-x_{0}\right)\left(A_{2} t\right)^{-1 / 3}$. Dividing the density $n$ by $\left(A_{2} t\right)^{-1 / 3}$, both of dimension $1 / L$, yields the second dimensionless quantity $H=\left(A_{2} t\right)^{1 / 3} n$.

[35] J. M. Burgers, The Nonlinear Diffusion Equation: Asymptotics, Solutions, and Statistical Problems (D. Reidel Publishing, Dordrecht, 1974).

[36] The shock wave described by Eq. (25), under the conservation 
condition Eq. (7), is completely determined by the four dimensional quantities $x-x_{1}, t, n$, and $A_{1}$. Again, it follows that there exists a description in terms of two dimensionless quantities. Now, $x-x_{1}$ and $t$ can be combined (together with $A_{1}$, whose dimension is $L^{2} / T$ ) into the dimensionless parameter $\eta=\left(x-x_{1}\right)\left(A_{1} t\right)^{-1 / 2}$. Dividing the density $n$ by $\left(A_{1} t\right)^{-1 / 2}$, which both have the same dimension $1 / L$, yields the second dimensionless quantity $G=\left(A_{1} t\right)^{1 / 2} n$.

[37] The same equation Eq. (23) with $\Gamma=0$ also happens to describe the decay of a vigorously shaken cluster in a nontilted array of compartments [26]. In that case, the fact that the parameter $\widetilde{B}_{L, R}$ does not appear in the equation is simply due to the strength of the shaking, since $\widetilde{B}_{L, R} \rightarrow 0$ for large values of the vibration amplitude $a$ and/or frequency $f$ [see Eq. (3)].

[38] J. Billingham and A. C. King, Wave Motion, Cambridge Texts in Applied Mathematics (Cambridge University Press, Cambridge, England, 2000).

[39] Note that also our earlier choice for $K=1000$ compartments was motivated by the diffusive stage, because only on a suffi- ciently long staircase do the particles get sufficiently diluted to bring the diffusion to life [22]. Now, to observe the selfsimilarity of the diffusion we need an even larger number of compartments.

[40] For $\gamma^{\prime}=20$, we compared the approximations in Eq. (38) to the numerical solutions of $R(n)=1$ for various $\widetilde{B}_{L}$ values. The approximation of $n_{\mathrm{i} \rightarrow \mathrm{ii}}$ was observed to become more accurate with increasing $\widetilde{B}_{L}$ and that of $n_{\mathrm{ii} \rightarrow \mathrm{iii}}$ less so. We found maximal deviations of about $20 \%$ but typically they were much smaller.

[41] In designing the example of Fig. 12, we dimensionalized $B_{L}$ such that if the system had contained only the large cluster, its motion would have been described by $B_{L}=1000$ (i.e., the value of choice throughout the paper). The addition of extra material in the form of the second cluster raises the total particle number $N_{\text {tot }}$ with a factor $3 / 2$, giving an increase of $B_{L}$ with a factor 9/4 [since $B_{L} \propto N_{\text {tot }}^{2}$, see Eq. (3)], so $B_{L}=2250$. 